\title{
Generalized kinetic and evolution equations in the approach of the nonequilibrium statistical operator
}

\author{
A. L. Kuzemsky \\ Bogoliubov Laboratory of Theoretical Physics, \\ Joint Institute for Nuclear Research, \\ 141980 Dubna, Moscow Region, Russid*
}

(Dated: September 7, 2018)

\begin{abstract}
The method of the nonequilibrium statistical operator developed by D. N. Zubarev is employed to analyse and derive generalized transport and kinetic equations. The degrees of freedom in solids can often be represented as a few interacting subsystems (electrons, spins, phonons, nuclear spins, etc.). Perturbation of one subsystem may produce a nonequilibrium state which is then relaxed to an equilibrium state due to the interaction between particles or with a thermal bath. The generalized kinetic equations were derived for a system weakly coupled to a thermal bath to elucidate the nature of transport and relaxation processes. It was shown that the "collision term" had the same functional form as for the generalized kinetic equations for the system with small interactions among particles. The applicability of the general formalism to physically relevant situations is investigated. It is shown that some known generalized kinetic equations (e.g. kinetic equation for magnons, Peierls equation for phonons) naturally emerges within the NSO formalism. The relaxation of a small dynamic subsystem in contact with a thermal bath is considered on the basis of the derived equations. The Schrodinger-type equation for the average amplitude describing the energy shift and damping of a particle in a thermal bath and the coupled kinetic equation describing the dynamic and statistical aspects of the motion are derived and analysed. The equations derived can help in the understanding of the origin of irreversible behavior in quantum phenomena.
\end{abstract}

PACS numbers: 05.20.Dd, 05.30.Ch, 05.60.-k, 05.60.Gg

Keywords: transport phenomena, method of the nonequilibrium statistical operator, system weakly coupled to a thermal bath, kinetic equations

\section{INTRODUCTION}

The aim of statistical mechanics is to give a consistent formalism for a microscopic description of macroscopic behavior of matter in bulk. The methods of equilibrium and nonequilibrium statistical mechanics have been fruitfully applied to a large variety of phenomena and materials [1, 2, 3, 4, 5, 6, 7]. The statistical mechanics of irreversible processes in solids, liquids, and complex materials like a soft matter are at the present time of much interest. The central problem of nonequilibrium statistical mechanics is to derive a set of equations which describe irreversible processes from the reversible equations of motion. The consistent calculation of transport coefficients is of particular interest because one can get information on the microscopic structure of the condensed matter. There exist a lot of theoretical methods for calculation of transport coefficients as a rule having a fairly restricted range of validity and applicability. The most extensively developed theory of transport processes is that based on the Boltzmann equation [8, 9]. However, this approach has strong restrictions and can reasonably be applied to a strongly rarefied gas of point particles [10]. For systems in the state of statistical equilibrium, there is the Gibbs distribution [11] by means of which it is possible to calculate an average value of any dynamical quantity. No such universal distribution has been formulated for irreversible processes. Thus, to proceed to the solution of problems of statistical mechanics of nonequilibrium systems, it is necessary to resort to various approximate methods [12, 13, 14, 15, 16, 17, 18]. Kubo and others 19, 20, 21] derived the quantum statistical expressions for transport coefficients such as electric and thermal conductivities. They considered the case of mechanical disturbances such as an electric field. The mechanical disturbance is expressed as a definite perturbing Hamiltonian and the deviation from equilibrium caused by it can be obtained by perturbation theory. On the other hand, thermal disturbances such as density and temperature gradients cannot be expressed as a perturbing Hamiltonian in an unambiguous way. During the last decades, a number of schemes have been concerned with a more general and consistent approach to transport theory [4, 22, 23, 24, 25, 26, 27, 28, 29, 30. These approaches, each in its own way, lead us to substantial advances in the understanding of the nonequilibrium behavior of many-particle classical and quantum systems. In addition, they have used dynamic

*Electronic address: kuzemsky@thsun1.jinr.ru 
arguments to obtain kinetic and balance equations which describe the irreversible evolution of a system from particular initial states. This field is very active and there are many aspects to the problem 31]. The purpose of the present work is to elucidate further the nature of transport processes and irreversible phenomena from a dynamic point of view. According to Montroll [32], "dynamics is the science of cleverly applying the operator $\exp (-i H t / \hbar)$ ". We wish to give a self-contained consideration of some general approach to the description of transport phenomena starting with dynamic equations. Our purpose here is to discuss the derivation, within the formalism of the nonequilibrium statistical operator [22, 27, 33], of the generalized transport and kinetic equations. On this basis we shall derive, by statistical mechanics methods, the kinetic equations for a system weakly coupled to a thermal bath.

In section [II we briefly review some basic concepts. In section IIA the derivation of the transport and kinetic equations within the NSO formalism is outlined. In section IIB we consider the application of the established equations to the derivation of the kinetic equations for magnons and phonons. Special attention is given to the problem of derivation of kinetic equations for a system weakly coupled to a thermal bath in section [II] On the basis of these equations the balance and master equations are obtained in section IV The behavior of a small dynamic system weakly coupled to a thermal bath is discussed in some detail in section $\mathbb{\nabla}$ The relaxation of a small dynamic subsystem in contact with a thermal bath is considered on the basis of the derived equations. The Schrodinger-type equation for an average amplitude describing the energy shift and damping of a particle in a thermal bath, and the coupled kinetic equation describing the dynamic and statistical aspects of the motion are derived and analysed in section VI

\section{OUTLINE OF THE NONEQUILIBRIUM STATISTICAL OPERATOR METHOD}

In this section, we briefly recapitulate the main ideas of the nonequilibrium statistical operator approach 22, 27, 33. for the sake of a self-contained formulation. The central statement of the statistical-mechanical picture is the fact that it is practically impossible to give a complete description of the state of a complex macroscopic system. We must substantially reduce the number of variables and confine ourselves to the description of the system which is considerably less then complete. The problem of predicting probable behavior of a system at some specified time is a statistical one. As it was shown by Gibbs 11] and Boltzmann [8], it is useful and workable to employ the technique of representing the system by means of an ensemble consisting of a large number of identical copies of a single system under consideration. The state of the ensemble is then described by a distribution function $\rho\left(\vec{r}_{1} \ldots \vec{r}_{n}, \vec{p}_{1} \ldots \vec{p}_{n}, t\right)$ in the phase space of a single system. This distribution function is chosen so that averages over the ensemble are in exact agreement with the incomplete ( macroscopic) knowledge of the state of the system at some specified time. Then the expected development of the system at subsequent times is modelled via the average behavior of members of the representative ensemble. It is evident that there are many different ways in which an ensemble could be constructed. As a result, the basic notion, the distribution function $\rho$ is not uniquely defined. Moreover, contrary to the description of a system in the state of thermodynamic equilibrium which is only one for fixed values of volume, energy, particle number, etc., the number of nonequilibrium states is large. The role of the relaxation times to equilibrium state was analysed in paper [34]. The precise definition of the nonequilibrium state is quite difficult and complicated, and is not uniquely specified. Since it is virtually impossible and impractical to try to describe in detail the state of a complex macroscopic system in the nonequilibrium state, the method of reducing the number of relevant variables was widely used. A large and important class of transport processes can reasonably be modelled in terms of a reduced number of macroscopic relevant variables 35. There are different time scales and different sets of the relevant variables [36, 37], e.g. hydrodynamic, kinetic, etc. This line of reasoning has led to seminal ideas on the construction of Gibbs-type ensembles for nonequilibrium systems [28, 38, 39, 40]. B. Robertson [41, 42, 43, 44] proposed the method of equations of motion for the "relevant" variables, the space- and time-dependent thermodynamic "coordinates" of a many-body nonequilibrium system which were derived directly from the Liouville equation. This was done by defining a generalized canonical density operator depending only upon present values of the thermodynamic "coordinates". The most satisfactory and workable approach to the construction of Gibbs-type ensembles for the nonequilibrium systems, as it appears to the writer, is the method of nonequilibrium statistical operator (NSO) developed by D. N. Zubarev [27]. The NSO method permits one to generalize the Gibbs ensemble method 11] to the nonequilibrium case and to construct a nonequilibrium statistical operator which enables one to obtain the transport equations and calculate the kinetic coefficients in terms of correlation functions, and which, in the case of equilibrium, goes over to the Gibbs distribution. Although this method is well known, we shall briefly recall it, mostly in order to introduce the notation needed in the following.

The NSO method sets out as follows. The irreversible processes which can be considered as a reaction of a system on mechanical perturbations can be analysed by means of the method of linear reaction on the external perturbation [19]. However, there is also a class of irreversible processes induced by thermal perturbations due to the internal inhomogeneity of a system. Among them we have, e.g., diffusion, thermal conductivity, and viscosity. In certain 
approximate schemes it is possible to express such processes by mechanical perturbations which artificially induce similar nonequilibrium processes. However, the fact is that the division of perturbations into mechanical and thermal ones is reasonable in the linear approximation only. In the higher approximations in the perturbation, mechanical perturbations can lead effectively to the appearance of thermal perturbations.

The NSO method permits one to formulate a workable scheme for description of the statistical mechanics of irreversible processes which include the thermal perturbation in a unified and coherent fashion. To perform this, it is necessary to construct statistical ensembles representing the macroscopic conditions determining the system. Such a formulation is quite reasonable if we consider our system for a suitable large time. For these large times the particular properties of the initial state of the system are irrelevant and the relevant number of variables necessary for description of the system reduces substantially [36].

As an introduction to the NSO method, let us describe the main ideas of this approach as follows. The basic hypothesis is that after small time-interval $\tau$ the nonequilibrium distribution is established. Moreover, it is supposed that it is weakly time-dependent by means of its parameter only. Then the statistical operator $\rho$ for $t \geq \tau$ can be considered as an "integral of motion" of the quantum Liouville equation

$$
\frac{\partial \rho}{\partial t}+\frac{1}{i \hbar}[\rho, H]=0
$$

Here $\frac{\partial \rho}{\partial t}$ denotes time differentiation with respect to the time variable on which the relevant parameters $F_{m}$ depend. It is important to note once again that $\rho$ depends on $t$ by means of $F_{m}(t)$ only. We may consider that the system is in thermal, material, and mechanical contact with a combination of thermal baths and reservoirs maintaining the given distribution of parameters $F_{m}$. For example, it can be the densities of energy, momentum, and particle number for the system which is macroscopically defined by given fields of temperature, chemical potential and velocity. It is assumed that the chosen set of parameters is sufficient to characterize macroscopically the state of the system. The set of the relevant parameters are dictated by the external conditions for the system under consideration and, therefore, the term $\frac{\partial \rho}{\partial t}$ appears as the result of the external influence upon the system. Due to this influence precisely, the behavior of the system is nonstationary.

In order to describe the nonequilibrium process, it is necessary also to choose the reduced set of relevant operators $P_{m}$, where $m$ is the index ( continuous or discrete). In the quantum case, all operators are considered to be in the Heisenberg representation

$$
P_{m}(t)=\exp \left(\frac{i H t}{\hbar}\right) P_{m} \exp \left(\frac{-i H t}{\hbar}\right)
$$

where $H$ does not depend on the time. The relevant operators may be scalars or vectors. The equations of motions for $P_{m}$ will lead to the suitable "evolution equations" [27]. In the quantum case

$$
\frac{\partial P_{m}(t)}{\partial t}+\frac{1}{i \hbar}\left[P_{m}(t), H\right]=0 .
$$

The time argument of the operator $P_{m}(t)$ denotes the Heisenberg representation with the Hamiltonian $H$ independent of time. Then we suppose that the state of the ensemble is described by a nonequilibrium statistical operator which is a functional of $P_{m}(t)$

$$
\rho(t)=\rho\left\{\ldots P_{m}(t) \ldots\right\}
$$

Then $\rho(t)$ satisfies the Liouville equation (11). Hence the quasi-equilibrium ("local-equilibrium") Gibbs-type distribution will have the form

$$
\rho_{q}=Q_{q}^{-1} \exp \left(-\sum_{m} F_{m}(t) P_{m}\right)
$$

where the parameters $F_{m}(t)$ have the sense of time-dependent thermodynamic parameters, e.g., of temperature, chemical potential, and velocity ( for the hydrodynamic stage), or the occupation numbers of one-particle states (for the kinetic stage). The statistical functional $Q_{q}$ is defined by demanding that the operator $\rho_{q}$ be normalized and equal to

$$
Q_{q}=\operatorname{Tr} \exp \left(-\sum_{m} F_{m}(t) P_{m}\right)
$$


This description is still very simplified. There are various effects which can make the picture more complicated. The quasi-equilibrium distribution is not necessarily close to the stationary stable state. There exists another, completely independent method for choosing a suitable quasi-equilibrium distribution [3, 4, 28, 45, 46]. For the state with the extremal value of the informational entropy [4, 28]

$$
S=-T r(\rho \ln \rho),
$$

provided that

$$
\operatorname{Tr}\left(\rho P_{m}\right)=<P_{m}>_{q} ; \quad \operatorname{Tr} \rho=1,
$$

it is possible to construct a suitable quasi-equilibrium ensemble. Then the corresponding quasi-equilibrium ( or local equilibrium ) distribution has the form

$$
\begin{array}{r}
\rho_{q}=\exp \left(\Omega-\sum_{m} F_{m}(t) P_{m}\right) \equiv \exp (S(t, 0)) \\
\Omega=\ln \operatorname{Tr} \exp \left(-\sum_{m} F_{m}(t) P_{m}\right)
\end{array}
$$

where $S(t, 0)$ can be called the entropy operator. The form of the quasi-equilibrium statistical operator was constructed in so as to ensure that the thermodynamic equalities for the relevant parameters $F_{m}(t)$

$$
\frac{\delta \ln Q_{q}}{\delta F_{m}(t)}=\frac{\delta \Omega}{\delta F_{m}(t)}=-<P_{m}>_{q} ; \quad \frac{\delta S}{\delta<P_{m}>_{q}}=F_{m}(t)
$$

are satisfied. It is clear that the variables $F_{m}(t)$ and $\left\langle P_{m}\right\rangle_{q}$ are thermodynamically conjugate. Here the notation used is $<\ldots\rangle_{q}=\operatorname{Tr}\left(\rho_{q} \ldots\right)$.

It is clear, however, that the operator $\rho_{q}$ itself does not satisfy the Liouville equation. The quasi-equilibrium operator should be modified in such a way that the resulting statistical operator satisfies the Liouville equation. This is the most delicate and subtle point of the whole method.

By definition a special set of operators should be constructed which depends on the time through the parameters $F_{m}(t)$ by taking the invariant part of the operators $F_{m}(t) P_{m}$ occurring in the logarithm of the quasi-equilibrium distribution, i.e.,

$$
\begin{array}{r}
B_{m}(t)=\overline{F_{m}(t) P_{m}}=\varepsilon \int_{-\infty}^{0} e^{\varepsilon t_{1}} F_{m}\left(t+t_{1}\right) P_{m}\left(t_{1}\right) d t_{1}= \\
F_{m}(t) P_{m}-\int_{-\infty}^{0} d t_{1} e^{\varepsilon t_{1}}\left(F_{m}\left(t+t_{1}\right) \dot{P}_{m}\left(t_{1}\right)+\dot{F}_{m}\left(t+t_{1}\right) P_{m}\left(t_{1}\right)\right)
\end{array}
$$

where $(\varepsilon \rightarrow 0)$ and

$$
\dot{P}_{m}=\frac{1}{i \hbar}\left[P_{m}, H\right] ; \quad \dot{F}_{m}(t)=\frac{d F_{m}(t)}{d t} .
$$

The parameter $\varepsilon>0$ will be set equal to zero, but only after the thermodynamic limit has been taken. Thus, the invariant part is taken with respect to the motion with Hamiltonian $H$. The operators $B_{m}(t)$ satisfy the Liouville equation in the limit $(\varepsilon \rightarrow 0)$

$$
\frac{\partial B_{m}}{\partial t}+\frac{1}{i \hbar}\left[B_{m}, H\right]=\varepsilon \int_{-\infty}^{0} d t_{1} e^{\varepsilon t_{1}}\left(F_{m}\left(t+t_{1}\right) \dot{P}_{m}\left(t_{1}\right)+\dot{F}_{m}\left(t+t_{1}\right) P_{m}\left(t_{1}\right)\right)
$$

The operation of taking the invariant part, of smoothing the oscillating terms, is used in the formal theory of scattering [47] to set the boundary conditions which exclude the advanced solutions of the Schrodinger equation [48]. It is most clearly seen when the parameters $F_{m}(t)$ are independent of time.

Differentiating $\overline{P_{m}}$ with respect to time gives

$$
\frac{\partial \overline{P_{m}(t)}}{\partial t}=\varepsilon \int_{-\infty}^{0} e^{\varepsilon t_{1}} \dot{P}_{m}\left(t+t_{1}\right) d t_{1}
$$


The $\overline{P_{m}(t)}$ will be called the integrals ( or quasi-integrals ) of motion, although they are conserved only in the limit $(\varepsilon \rightarrow 0)$. It is clear that for the Schrodinger equation such a procedure excludes the advanced solutions by choosing the initial conditions. In the present context this procedure leads to the selection of the retarded solutions of the Liouville equation. This philosophy has been pressed by the necessity of a consistent description of the irreversibility which is, according to [49], " at once a profound and an elusive concept" ( c.f., a discussion in Refs. [31, 50]).

It should be noted that the same calculations can also be made with a deeper concept, the methods of quasiaverages [27, 33, 51]. Let us note once again that the quantum Liouville equation, like the classical one, is symmetric under time-reversal transformation. However, the solution of the Liouville equation is unstable with respect to small perturbations violating this symmetry of the equation. Indeed, let us consider the Liouville equation with an infinitesimally small source into the right-hand side

$$
\frac{\partial \rho_{\varepsilon}}{\partial t}+\frac{1}{i \hbar}\left[\rho_{\varepsilon}, H\right]=-\varepsilon\left(\rho_{\varepsilon}-\rho_{q}\right)
$$

or equivalently

$$
\frac{\partial \ln \rho_{\varepsilon}}{\partial t}+\frac{1}{i \hbar}\left[\ln \rho_{\varepsilon}, H\right]=-\varepsilon\left(\ln \rho_{\varepsilon}-\ln \rho_{q}\right)
$$

where $(\varepsilon \rightarrow 0)$ after the thermodynamic limit. This equation (14) is analogous to the corresponding equation of the quantum scattering theory [47, 48]. The introduction of infinitesimally small sources into the Liouville equation is equivalent to the boundary condition

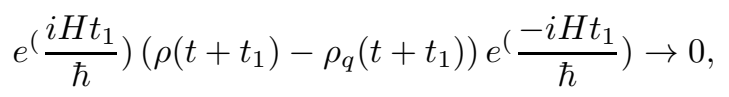

where $t_{1} \rightarrow-\infty$ after the thermodynamic limiting process. It was shown [27, 33] that the operator $\rho_{\varepsilon}$ has the form

$$
\rho_{\varepsilon}(t, t)=\varepsilon \int_{-\infty}^{t} d t_{1} e^{\varepsilon\left(t_{1}-t\right)} \rho_{q}\left(t_{1}, t_{1}\right)=\varepsilon \int_{-\infty}^{0} d t_{1} e^{\varepsilon t_{1}} \rho_{q}\left(t+t_{1}, t+t_{1}\right)
$$

Here the first argument of $\rho(t, t)$ is due to the indirect time-dependence via the parameters $F_{m}(t)$ and the second one is due to the Heisenberg representation. The required nonequilibrium statistical operator is defined as

$$
\rho_{\varepsilon}=\rho_{\varepsilon}(t, 0)=\overline{\rho_{q}(t, 0)}=\varepsilon \int_{-\infty}^{0} d t_{1} e^{\varepsilon t_{1}} \rho_{q}\left(t+t_{1}, t_{1}\right)
$$

Hence the nonequilibrium statistical operator can then be written in the form

$$
\begin{gathered}
\rho=Q^{-1} \exp \left(-\sum_{m} B_{m}\right)=Q^{-1} \exp \left(-\sum_{m} \varepsilon \int_{-\infty}^{0} d t_{1} e^{\varepsilon t_{1}}\left(F_{m}\left(t+t_{1}\right) P_{m}\left(t_{1}\right)\right)\right)= \\
Q^{-1} \exp \left(-\sum_{m} F_{m}(t) P_{m}+\sum_{m} \int_{-\infty}^{0} d t_{1} e^{\varepsilon t_{1}}\left[\dot{F}_{m}\left(t+t_{1}\right) P_{m}\left(t_{1}\right)+F_{m}\left(t+t_{1}\right) \dot{P}_{m}\left(t_{1}\right)\right]\right)
\end{gathered}
$$

Let us write down Eq. (15) in the following form:

$$
\frac{d}{d t}\left(e^{\varepsilon t} \ln \rho(t, t)\right)=\varepsilon e^{\varepsilon t} \ln \rho_{q}(t, t)
$$

where

$$
\ln \rho(t, t)=U^{\dagger}(t, 0) \ln \rho(t, 0) U(t, 0) ; \quad U(t, 0)=\exp \left(\frac{i H t}{\hbar}\right)
$$

After integration, Eq.(20), over the interval $(-\infty, 0)$ we get

$$
\ln \rho(t, t)=\varepsilon \int_{-\infty}^{0} d t_{1} e^{\varepsilon t_{1}} \ln \rho_{q}\left(t+t_{1}, t+t_{1}\right)
$$

Here we suppose that $\lim _{\varepsilon \rightarrow 0^{+}} \ln \rho(t, t)=0$.

Now we can rewrite the nonequilibrium statistical operator in the following useful form:

$$
\rho(t, 0)=\exp \left(-\varepsilon \int_{-\infty}^{0} d t_{1} e^{\varepsilon t_{1}} \ln \rho_{q}\left(t+t_{1}, t_{1}\right)\right)=\exp \overline{\left(\ln \rho_{q}(t, 0)\right)} \equiv \exp \overline{(-S(t, 0))}
$$


The average value of any dynamic variable $A$ is given by

$$
<A>=\lim _{\varepsilon \rightarrow 0^{+}} \operatorname{Tr}(\rho(t, 0) A)
$$

and is, in fact, the quasi-average. The normalization of the quasi-equilibrium distribution $\rho_{q}$ will persists after taking the invariant part if the following conditions are required

$$
\operatorname{Tr}\left(\rho(t, 0) P_{m}\right)=<P_{m}>=<P_{m}>_{q} ; \quad \operatorname{Tr} \rho=1
$$

Before closing this section, we shall mention some modification of the "canonical" NSO method which was proposed in [46] and which one has to take into account in a more accurate treatment of transport processes.

\section{A. The Transport and Kinetic Equations}

It is well known that the kinetic equations are of great interest in the theory of transport processes. Indeed, as it was shown in the preceding section, the main quantities involved are the following thermodynamically conjugate values:

$$
<P_{m}>=-\frac{\delta \Omega}{\delta F_{m}(t)} ; \quad F_{m}(t)=\frac{\delta S}{\delta<P_{m}>}
$$

The generalized transport equations which describe the time evolution of variables $\left\langle P_{m}\right\rangle$ and $F_{m}$ follow from the equation of motion for the $P_{m}$, averaged with the nonequilibrium statistical operator (23). It reads

$$
<\dot{P}_{m}>=-\sum_{n} \frac{\delta^{2} \Omega}{\delta F_{m}(t) \delta F_{n}(t)} \dot{F}_{n}(t) ; \quad \dot{F}_{m}(t)=\sum_{n} \frac{\delta^{2} S}{\delta<P_{m}>\delta<P_{n}>}<\dot{P}_{n}>
$$

The entropy production has the form

$$
\dot{S}(t)=<\dot{S}(t, 0)>=-\sum_{m}<\dot{P}_{m}>F_{m}(t)=-\sum_{n, m} \frac{\delta^{2} \Omega}{\delta F_{m}(t) \delta F_{n}(t)} \dot{F}_{n}(t) F_{m}(t)
$$

These equations are the mutually conjugate and with Eq.(26) form a complete system of equations for the calculation of values $<P_{m}>$ and $F_{m}$.

Let us illustrate the NSO method by considering the derivation of kinetic equations for a system of weakly interacting particles [52]. In this case the Hamiltonian can be written in the form

$$
H=H_{0}+V
$$

where $H_{0}$ is the Hamiltonian of noninteracting particles ( or quasiparticles ) and $V$ is the operator describing the weak interaction among them. Let us choose the set of operators $P_{m}=P_{k}$ whose average values correspond to the particle distribution functions, e.g., $a_{k}^{\dagger} a_{k}$ or $a_{k}^{\dagger} a_{k+q}$. Here $a_{k}^{\dagger}$ and $a_{k}$ are the creation and annihilation second quantized operators ( Bose or Fermi type). These operators obey the following quantum equation of motion:

$$
\dot{P}_{k}=\frac{1}{i \hbar}\left[P_{k}, H\right]
$$

It is reasonable to assume that the following relation is fulfilled

$$
\left[P_{k}, H_{0}\right]=\sum_{l} c_{k l} P_{l},
$$

where $c_{k l}$ are some coefficients ( c-numbers).

According to Eq.(19), the nonequilibrium statistical operator has the form

$$
\rho=Q^{-1} \exp \left(-\sum_{k} F_{k}(t) P_{k}+\sum_{k} \int_{-\infty}^{0} d t_{1} e^{\varepsilon t_{1}}\left[\dot{F}_{k}\left(t+t_{1}\right) P_{k}\left(t_{1}\right)+F_{k}\left(t+t_{1}\right) \dot{P}_{k}\left(t_{1}\right)\right]\right)
$$


After elimination of the time-derivatives with the help of the equation $<P_{k}>=<P_{k}>_{q}$ it can be shown 52 that the integral term in the exponent, Eq.(32), will be proportional to the interaction $V$. The averaging of Eq.(30) with NSO (32) gives the generalized kinetic equations for $\left\langle P_{k}\right\rangle$

$$
\frac{d<P_{k}>}{d t}=\frac{1}{i \hbar}<\left[P_{k}, H\right]>=\frac{1}{i \hbar} \sum_{l} c_{k l}<P_{l}>+\frac{1}{i \hbar}<\left[P_{k}, V\right]>
$$

Hence the calculation of the r.h.s. of (33) leads to the explicit expressions for the "collision integral" ( collision terms). Since the interaction is small, it is possible to rewrite Eq. (33) in the following form:

$$
\frac{d<P_{k}>}{d t}=L_{k}^{0}+L_{k}^{1}+L_{k}^{21}+L_{k}^{22}
$$

where

$$
\begin{gathered}
L_{k}^{0}=\frac{1}{i \hbar} \sum_{l} c_{k l}<P_{l}>_{q} \\
L_{k}^{1}=\frac{1}{i \hbar}<\left[P_{k}, V\right]>_{q} \\
L_{k}^{21}=\frac{1}{\hbar^{2}} \int_{-\infty}^{0} d t_{1} e^{\varepsilon t_{1}}<\left[V\left(t_{1}\right),\left[P_{k}, V\right]\right]>_{q} \\
L_{k}^{22}=\frac{1}{\hbar^{2}} \int_{-\infty}^{0} d t_{1} e^{\varepsilon t_{1}}<\left[V\left(t_{1}\right), i \hbar \sum_{l} P_{l} \frac{\partial L_{k}^{1}\left(\ldots<P_{l}>\ldots\right)}{\partial<P_{l}>}\right]>_{q}
\end{gathered}
$$

The higher order terms proportional to the $V^{3}, V^{4}$, etc., can be derived straightforwardly.

\section{B. Kinetic Equations for Magnons and Phonons}

The dynamic behavior of charge [53], magnetic [54], and lattice 55] systems is of interest for the study of transport processes in solids. Partial emphasis has been placed on the derivation of the kinetic equations describing the hot electron transport in semiconductors [56, 57], and the relaxation of magnons [58, 59] and phonons [55, 60] due to the inelastic scattering of quasiparticles.

We discuss briefly in this section the processes occurring after the switching off the external magnetic field in a ferromagnetic crystal. Our main interest is in ferromagnetic insulators, where the dominant interaction is the Heisenberg exchange coupling $J \vec{S}_{i} \vec{S}_{j}$. It is well known that a strong microwave magnetic field applied parallel to the dc field can give rise to parametric excitation of spin waves [58, 61, 62]. In this technique the wave number of the potentially unstable spin waves can be changed by varying the dc magnetic field. One thus obtains information about the variation of the spin-wave relaxation time with the wave number 63, 64, 65, 66, 67. Because of its relative simplicity the "parallel pumping" technique has proved very useful in determining rather fundamental properties of ferromagnetic materials. The subharmonic generation of spin waves at high power levels is an efficient research tool for probing magnon-magnon and magnon-phonon interactions. Useful information about the spin-wave relaxation rate can be deduced from the kinetic equations to study magnon-magnon and magnon-phonon interactions.

Here the spin-wave relaxation processes arising from the dipolar interaction will be considered as an example. The Hamiltonian has the form

$$
H=-\frac{1}{2} \sum_{l \neq l^{\prime}} J\left(R_{l l^{\prime}}\right) \overrightarrow{S_{l}} \overrightarrow{S_{l^{\prime}}}+2 \mu_{0} h_{0} \sum_{l} S_{l}^{z}+2 \mu_{0}^{2} \sum_{l \neq l^{\prime}} \frac{1}{R_{l l^{\prime}}^{5}}\left(R_{l l^{\prime}}^{2} \overrightarrow{S_{l}} \overrightarrow{S_{l^{\prime}}}-3\left(\overrightarrow{R_{l l^{\prime}}} \overrightarrow{S_{l^{\prime}}}\right)\left(\overrightarrow{R_{l l^{\prime}}} \overrightarrow{S_{l^{\prime}}}\right)\right)
$$

This Hamiltonian contains Zeeman energy, exchange energy, and dipolar energy. To treat this Hamiltonian, it should be expressed in terms of the amplitudes of the normal modes or spin waves 68 . The amplitudes of the normal modes are quantum-mechanically interpreted as creation and annihilation operators ( usually bosons). We get [68]

$$
S_{l}^{+}=S_{l}^{x}+i S_{l}^{y}=\sqrt{2 S} b_{l}^{\dagger} \sqrt{1-\frac{b_{l}^{\dagger} b_{l}}{2 S}} ; \quad S_{l}^{-}=S_{l}^{x}-i S_{l}^{y}=\sqrt{2 S} \sqrt{1-\frac{b_{l}^{\dagger} b_{l}}{2 S} b_{l}} ; \quad S_{l}^{z}=-S+b_{l}^{\dagger} b_{l}
$$


We adopt the notation

$$
b_{i}=N^{-1 / 2} \sum_{\vec{k}} b_{k} \exp \left(i \vec{k} \vec{R}_{i}\right), \quad b_{i}^{\dagger}=N^{-1 / 2} \sum_{\vec{k}} b_{k}^{\dagger} \exp \left(-i \vec{k} \vec{R}_{i}\right)
$$

The transformed Hamiltonian contains a term that is quadratic in the spin-wave amplitudes $H^{(2)}$ and also terms that are of higher order, $H^{(3)}, H^{(4)}$, etc.

$$
H=H^{(2)}+H^{(3)}+H^{(4)}+\ldots
$$

The eigenstates of the quadratic part of the Hamiltonian $H^{(2)}$ can be characterized by the occupation numbers $c_{k}^{\dagger} c_{k}$, i.e., the quadratic part can be diagonalized to the form [68]

$$
H^{(2)}=\sum_{k} \epsilon(k) c_{k}^{\dagger} c_{k} ; \quad \epsilon(k)=\hbar \omega(k)
$$

Here the operators $c_{k}^{\dagger}$ and $c_{k}$ are the second-quantized operators of creation and annihilation of magnons. All higher order terms in the Hamiltonian lead to transitions between the eigenstates. In terms of the magnon operators these terms are given by

$$
\begin{gathered}
H^{(3)}=\sum_{k p p^{\prime}} \Phi\left(k, p, p^{\prime}\right) c_{k}^{\dagger} c_{p}^{\dagger} c_{p^{\prime}} \Delta\left(\vec{k}+\vec{p}-\overrightarrow{p^{\prime}}\right)+H . C . \\
H^{(4)}=\sum_{k p p^{\prime} r} \Phi\left(k, p ; p^{\prime}, r\right) c_{k}^{\dagger} c_{p}^{\dagger} c_{p^{\prime}} c_{r} \Delta\left(\vec{k}+\vec{p}-\overrightarrow{p^{\prime}}-\vec{r}\right)+H . C .
\end{gathered}
$$

Usually, only that term in the Hamiltonian which is of the third order in the amplitudes of the normal modes is considered explicitly, because only this term leads to the relaxation rates proportional to the temperature in the high-temperature limit.

Let us apply now the formalism of generalized kinetic equations, as described above. We suppose that the set of averages $\left\langle P_{k}\right\rangle=\left\langle c_{k}^{\dagger} c_{k}\right\rangle=\left\langle n_{k}\right\rangle$ characterize the nonequilibrium state of the system. The quasi-equilibrium statistical operator has the form

$$
\rho_{q}=Q_{q}^{-1} \exp \left(-\sum_{k} F_{k}(t) n_{k}\right) ; \quad Q_{q}^{-1}=\operatorname{Tr} \exp \left(-\sum_{k} F_{k}(t) n_{k}\right)
$$

The kinetic equation (34) can then be expressed by

$$
\frac{d<n_{k}>}{d t}=L_{k}^{0}+L_{k}^{1}+L_{k}^{21}+L_{k}^{22}
$$

For both the contributions $H^{(3)}$ and $H^{(4)}$ the following equality holds:

$$
L_{k}^{0}=L_{k}^{1}=L_{k}^{22}=0
$$

Let us first consider the contribution of the term $H^{(3)}$. We can then write

$$
\begin{array}{r}
L_{k}^{21}=-\frac{8 \pi}{\hbar} \sum_{\vec{p}, \vec{p}^{\prime}}\left\{\left|\Phi\left(k, p, p^{\prime}\right)\right|^{2} \delta\left(\omega(k)+\omega(p)-\omega\left(p^{\prime}\right)\right) \Delta\left(\vec{k}+\vec{p}-\overrightarrow{p^{\prime}}\right)\right. \\
{\left[\left(<n_{k}>+1\right)\left(<n_{p}>+1\right)<n_{p^{\prime}}>-<n_{k}><n_{p}>\left(<n_{p^{\prime}}>+1\right)\right]} \\
-\frac{1}{2}\left|\Phi\left(k, p, p^{\prime}\right)\right|^{2} \delta\left(\omega(k)-\omega(p)-\omega\left(p^{\prime}\right)\right) \Delta\left(\vec{k}-\vec{p}-\overrightarrow{p^{\prime}}\right) \\
{\left[\left(<n_{k}>+1\right)<n_{p}><n_{p^{\prime}}>-<n_{k}>\left(<n_{p}>+1\right)\left(<n_{p^{\prime}}>+1\right)\right]}
\end{array}
$$

We can make the same calculation to obtain $L_{k}^{21}$ for the magnon-magnon scattering term $H^{(4)}$

$$
\begin{array}{r}
L_{k}^{21}=-\frac{16 \pi}{\hbar} \sum_{\vec{p}, \vec{p}^{\prime}, \vec{r}}\left\{\left|\Phi\left(k, p, p^{\prime}, r\right)\right|^{2} \delta\left(\omega(k)+\omega(p)-\omega\left(p^{\prime}\right)-\omega(r)\right) \Delta\left(\vec{k}+\vec{p}-\overrightarrow{p^{\prime}}-\vec{r}\right)\right. \\
{\left[\left(<n_{k}>+1\right)\left(<n_{p}>+1\right)<n_{p^{\prime}}><n_{r}>-<n_{k}><n_{p}>\left(<n_{p^{\prime}}>+1\right)\left(<n_{r}>+1\right)\right]}
\end{array}
$$


Here the notation was used

$$
<n_{k}>=N(\hbar \omega(k))=[\exp (\beta \hbar \omega(k))-1]^{-1}
$$

The quantities $\Phi\left(k, p, p^{\prime}\right)$ and $\Phi\left(k, p, p^{\prime}, r\right)$ are the combination of the matrix elements which describe the various transitions between spin eigenstates [58]. Equation (48) corresponds precisely to the rate equation which describes the change of the average occupation number $\left\langle n_{k}\right\rangle$ of the mode $k$ derived in [58]. The discussion of the two relevant relaxation rates $\tau_{k}^{-1}$ ( due to the confluence and splitting) is given there. The types of kinetic equations, Eqs. (48), (49), involved in our derivation and the conclusions arrived at show very clearly that the NSO method is a workable and useful approach for derivation of the kinetic equations for concrete physical problems. As far as the kinetic equations for magnons is concerned, its convenience can become even more evident if one needs to take into account higher order magnon processes ( four, five, etc.). The higher order processes may give rise to additional and unusual behavior ( i.e., a general heating of the spin-wave system causing the saturation, additional smaller peaks and kinks in the measured curves, etc.)

It is evident that a similar derivation can be given for the kinetic equation for phonons. The theory of thermal conductivity [60, 69] has been extensively developed beginning with the kinetic theory of Peierls [9, 55, 70]. The theory of lattice thermal conductivity invented by Peierls [55, 70] is based on the assumption that the perturbing mechanisms to the harmonic case ( anharmonicity, imperfections) are small in magnitude. The Peierls collision term for the three-phonon processes $H^{(3)}$ looks like

$$
\begin{array}{r}
L_{k}^{21} \sim \frac{a \pi}{\hbar} \sum_{\vec{p}, \vec{p}^{\prime}}\left\{\left|\Phi\left(k, p, p^{\prime}\right)\right|^{2} \delta\left(\omega(k)+\omega(p)-\omega\left(p^{\prime}\right)\right)\right. \\
{\left[\left(<n_{k}>+1\right)\left(<n_{p}>+1\right)<n_{p^{\prime}}>-<n_{k}><n_{p}>\left(<n_{p^{\prime}}>+1\right)\right]} \\
+\frac{1}{2}\left|\Phi\left(k, p, p^{\prime}\right)\right|^{2} \delta\left(\omega(k)-\omega(p)-\omega\left(p^{\prime}\right)\right)\left[\left(<n_{k}>+1\right)<n_{p}><n_{p^{\prime}}>\right]
\end{array}
$$

Note that our calculations show that three- and four-phonon processes behave quite differently. One expects that the stronger the anharmonicity the larger the thermal resistance. To catch this trend, some sophisticated formalisms [71] have been developed which utilize a modified version of the Peierls-Boltzmann equation. A useful approach to improving the initial Peierls theory corresponds to the derivation of a generalized Peierls-Boltzmann equation, where the phonons in the collision term are treated not as free phonons but as quasiparticles with a finite width and damping which are determined self-consistently. Crystal lattices at low temperatures represent an interacting system of quasiparticles in which we observe two relaxation mechanisms of widely different time scales, i.e., the system either at short or long times after its initial perturbation from equilibrium. For the long-time behavior of the system it is possible to formulate the problem in terms of the correlation functions of quantities relaxing slowly, such as densities of conserved variables in the system. The corresponding transport equations are similar in structure to the phonon Boltzmann equation with a modification of the collision term. A detailed study of the transport equations for phonon systems is not within the scope of this paper and deserves a separate consideration.

\section{SYSTEM IN THERMAL BATH: GENERALIZED KINETIC EQUATIONS}

We now proceed to derive generalized kinetic equations for the system weakly coupled to a thermal bath. Examples of such systems can be an atomic ( or molecular) system interacting with the electromagnetic field it generates as with a thermal bath, a system of electrons or exitons interacting with the phonon field, etc. Our aim is to investigate relaxation processes in two weakly interacting subsystems, one of which is in the nonequilibrium state and the other is considered as a thermal bath. The concept of thermal bath or heat reservoir, i.e., a system that has effectively an infinite number of degrees of freedom, was not formulated precisely. A standard definition of the thermal bath is a heat reservoir defining a temperature of the system environment. From a mathematical point of view [37], a heat bath is something that gives a stochastic influence on the system under consideration. In this sense, the generalized master equation 72,73 is a tool for extracting the dynamics of a subsystem of a larger system by the use of a special projection techniques [4]. The problem of a small system weakly interacting with a heat reservoir has various aspects. For example, a useful model of the lattice thermal conduction is a problem of a stationary energy current through a crystalline lattice in contact with external heat reservoirs 75, 76, 77]. Basic to the derivation of a transport equation for a small system weakly interacting with a heat bath is a proper introduction of model assumptions.

We are interested here in the problem of derivation of the kinetic equations for a certain set of average values ( occupation numbers, spins, etc.) which characterize the nonequilibrium state of the system.

Let us consider the relaxation of a small subsystem weakly interacting with a thermal bath. The Hamiltonian of the 
total system is taken in the following form:

$$
H=H_{1}+H_{2}+V
$$

where

$$
H_{1}=\sum_{\alpha} E_{\alpha} a_{\alpha}^{\dagger} a_{\alpha} ; \quad V=\sum_{\alpha, \beta} \Phi_{\alpha \beta} a_{\alpha}^{\dagger} a_{\beta}, \quad \Phi_{\alpha \beta}=\Phi_{\alpha \beta}^{\dagger}
$$

Here $H_{1}$ is the Hamiltonian of the small subsystem, and $a_{\alpha}^{\dagger}$ and $a_{\alpha}$ are the creation and annihilation second quantized operators of quasiparticles in the small subsystem with energies $E_{\alpha}, V$ is the operator of the interaction between the small subsystem and the thermal bath, and $H_{2}$ is the Hamiltonian of the thermal bath which we do not write explicitly. The quantities $\Phi_{\alpha \beta}$ are the operators acting on the thermal bath variables.

We are interested in the kinetic stage of the nonequilibrium process in the system weakly coupled to a thermal bath. Therefore, we assume that the state of this system is determined completely by the set of averages $\left.<P_{\alpha \beta}\right\rangle=\left\langle a_{\alpha}^{\dagger} a_{\beta}\right\rangle$ and the state of the thermal bath by $\left\langle H_{2}\right\rangle$, where $\langle\ldots\rangle$ denotes the statistical average with the nonequilibrium statistical operator, which will be defined below.

In order to pursue our discussion, we will use the whole development in section [II We take the quasi-equilibrium statistical operator $\rho_{q}$ in the form

$$
\begin{array}{r}
\rho_{q}(t)=\exp (-S(t, 0)), \quad S(t, 0)=\Omega(t)+\sum_{\alpha \beta} P_{\alpha \beta} F_{\alpha \beta}(t)+\beta H_{2} \\
\Omega=\ln \operatorname{Tr} \exp \left(-\sum_{\alpha \beta} P_{\alpha \beta} F_{\alpha \beta}(t)-\beta H_{2}\right)
\end{array}
$$

Here $F_{\alpha \beta}(t)$ are the thermodynamic parameters conjugated with $P_{\alpha \beta}$, and $\beta$ is the reciprocal temperature of the thermal bath. All the operators are considered in the Heisenberg representation. The nonequilibrium statistical operator has the form

$$
\begin{array}{r}
\rho(t)=\exp (-\overline{S(t, 0)}), \\
\overline{S(t, 0)}=\varepsilon \int_{-\infty}^{0} d t_{1} e^{\varepsilon t_{1}}\left(\Omega\left(t+t_{1}\right)+\sum_{\alpha \beta} P_{\alpha \beta} F_{\alpha \beta}(t)+\beta H_{2}\right)
\end{array}
$$

The parameters $F_{\alpha \beta}(t)$ are determined from the condition $\left\langle P_{\alpha \beta}>=<P_{\alpha \beta}>_{q}\right.$.

In the derivation of the kinetic equations we use the perturbation theory in a "weakness of interaction" and assume that the equality $\left\langle\Phi_{\alpha \beta}\right\rangle_{q}=0$ holds, while other terms can be added to the renormalized energy of the subsystem. The nonequilibrium statistical operator can be rewritten as

$$
\begin{array}{r}
\rho(t)=Q^{-1} \exp (-L(t)), \\
L(t)=\varepsilon \int_{-\infty}^{0} d t_{1} e^{\varepsilon t_{1}}\left(\sum_{\alpha \beta} P_{\alpha \beta} F_{\alpha \beta}\left(t+t_{1}\right)+\beta H_{2}\left(t_{1}\right)\right)
\end{array}
$$

Integrating in Eq.(55) by parts, we obtain

$$
\begin{array}{r}
L(t)=\sum_{\alpha \beta} P_{\alpha \beta} F_{\alpha \beta}(t)+\beta H_{2} \\
-\int_{-\infty}^{0} d t_{1} e^{\varepsilon t_{1}}\left(\sum_{\alpha \beta} \dot{P}_{\alpha \beta}\left(t_{1}\right) F_{\alpha \beta}\left(t+t_{1}\right)+\sum_{\alpha \beta} P_{\alpha \beta}\left(t_{1}\right) \frac{\partial F_{\alpha \beta}\left(t+t_{1}\right)}{\partial t_{1}}+\beta \dot{H}_{2}\left(t_{1}\right)\right)
\end{array}
$$

For further considerations it is convenient to rewrite $\rho_{q}$ as

$$
\rho_{q}=\rho_{1} \rho_{2}=Q_{q}^{-1} \exp \left(-L_{0}(t)\right),
$$

where

$$
\rho_{1}=Q_{1}^{-1} \exp \left(-\sum_{\alpha \beta} P_{\alpha \beta} F_{\alpha \beta}(t)\right) ; \quad Q_{1}=\operatorname{Tr} \exp \left(-\sum_{\alpha \beta} P_{\alpha \beta} F_{\alpha \beta}(t)\right)
$$




$$
\begin{gathered}
\rho_{2}=Q_{2}^{-1} e^{-\beta H_{2}} ; \quad Q_{2}=\operatorname{Tr} \exp \left(-\beta H_{2}\right) \\
Q_{q}=Q_{1} Q_{2} ; \quad L_{0}=\sum_{\alpha \beta} P_{\alpha \beta} F_{\alpha \beta}(t)+\beta H_{2}
\end{gathered}
$$

We now turn to the derivation of the kinetic equations. The starting point is the kinetic equations in the following implicit form:

$$
\frac{d<P_{\alpha \beta}>}{d t}=\frac{1}{i \hbar}<\left[P_{\alpha \beta}, H\right]>=\frac{1}{i \hbar}\left(E_{\beta}-E_{\alpha}\right)<P_{\alpha \beta}>+\frac{1}{i \hbar}<\left[P_{\alpha \beta}, V\right]>
$$

We restrict ourselves to the second-order in powers of $V$ in calculating the r.h.s. of (61). To this end, we must obtain $\rho(t)$ in the first-order in $V$. We get

$$
\begin{array}{r}
\frac{\partial F_{\alpha \beta}\left(t+t_{1}\right)}{\partial t_{1}}=\frac{i}{\hbar}\left(E_{\beta}-E_{\alpha}\right) F_{\alpha \beta}\left(t+t_{1}\right)-\sum_{\mu \nu} \frac{\partial F_{\alpha \beta}\left(t+t_{1}\right)}{\partial<P_{\mu \nu}>} \frac{1}{i \hbar}<\left[P_{\mu \nu}\left(t_{1}\right), V\left(t_{1}\right)\right]>= \\
\frac{i}{\hbar}\left(E_{\beta}-E_{\alpha}\right) F_{\alpha \beta}\left(t+t_{1}\right)-\sum_{\mu \nu \gamma} \frac{\partial F_{\alpha \beta}\left(t+t_{1}\right)}{\partial<P_{\mu \nu}>}\left(<\Phi_{\nu \gamma} P_{\mu \gamma}>-<\Phi_{\gamma \mu} P_{\gamma \nu}>\right)
\end{array}
$$

Restricting ourselves to the linear terms in Eq. (62), we obtain

$$
\begin{array}{r}
\frac{\partial F_{\alpha \beta}\left(t+t_{1}\right)}{\partial t_{1}} \simeq \frac{i}{\hbar}\left(E_{\beta}-E_{\alpha}\right) F_{\alpha \beta}\left(t+t_{1}\right)-\frac{1}{i \hbar} \sum_{\mu \nu} \frac{\partial F_{\alpha \beta}\left(t+t_{1}\right)}{\partial<P_{\mu \nu}>}\left(<\Phi_{\nu \gamma}>_{q}<P_{\mu \gamma}>-<\Phi_{\gamma \mu}>_{q}<P_{\gamma \nu}>\right) \\
=\frac{i}{\hbar}\left(E_{\beta}-E_{\alpha}\right) F_{\alpha \beta}\left(t+t_{1}\right)
\end{array}
$$

The quantities, $\dot{P}_{\alpha \beta}\left(t_{1}\right)$ and $\dot{H}_{2}\left(t_{1}\right)$ in the first-order in interaction have the form

$$
\begin{array}{r}
\dot{P}_{\alpha \beta}\left(t_{1}\right)=\frac{1}{i \hbar}\left(E_{\beta}-E_{\alpha}\right) P_{\alpha \beta}\left(t_{1}\right)+\frac{1}{i \hbar}\left[P_{\alpha \beta}, V\left(t_{1}\right)\right] \\
\dot{H}_{2}\left(t_{1}\right)=\frac{1}{i \hbar}\left[H_{2}\left(t_{1}\right), V\left(t_{1}\right)\right]
\end{array}
$$

Here and below all the operators are taken in the interaction representation. Using Eqs. (63) and (64) we find

$$
L(t)=L_{0}-\int_{-\infty}^{0} d t_{1} e^{\varepsilon t_{1}}\left[\sum_{\alpha \beta} P_{\alpha \beta}\left(t_{1}\right) F_{\alpha \beta}\left(t+t_{1}\right)+\beta H_{2}\left(t_{1}\right), V\left(t_{1}\right)\right]
$$

It can be verified that the expression $\sum_{\alpha \beta} P_{\alpha \beta}\left(t_{1}\right) F_{\alpha \beta}\left(t+t_{1}\right)+\beta H_{2}$ is independent of $t_{1}$ in the zero-order in interaction and consequently is equal to $L_{0}$. Then for $\rho(t)$ in the linear approximation in interaction $V$ we have

$$
\rho(t)=\rho_{q}-\frac{i}{\hbar} \rho_{q} \int_{-\infty}^{0} d t_{1} e^{\varepsilon t_{1}} \int_{0}^{1} d \lambda e^{\lambda L_{0}}\left[L_{0}, V\left(t_{1}\right)\right] e^{-\lambda L_{0}}
$$

By integrating in Eq.(66) over $\lambda$ and using the relation

$$
e^{\lambda L_{0}}\left[L_{0}, V\left(t_{1}\right)\right] e^{-\lambda L_{0}}=\frac{d}{d \lambda} e^{\lambda L_{0}} V\left(t_{1}\right) e^{-\lambda L_{0}}
$$

we get

$$
\rho(t)=\rho_{q}-\frac{i}{\hbar} \int_{-\infty}^{0} d t_{1} e^{\varepsilon t_{1}}\left[V\left(t_{1}\right), \rho_{q}\right]
$$

Finally, with the aid of Eq. (68) we obtain the kinetic equations for $\left\langle P_{\alpha \beta}\right\rangle$ in the form

$$
\frac{d<P_{\alpha \beta}>}{d t}=\frac{1}{i \hbar}\left(E_{\beta}-E_{\alpha}\right)<P_{\alpha \beta}>-\frac{1}{\hbar^{2}} \int_{-\infty}^{0} d t_{1} e^{\varepsilon t_{1}}<\left[\left[P_{\alpha \beta}, V\right], V\left(t_{1}\right)\right]>_{q}
$$


The last term of the right-hand side of Eq. (69) can be called the generalized "collision integral". Thus, we can see that the collision term for the system weakly coupled to the thermal bath has a convenient form of the double commutator as for the generalized kinetic equations (37) for the system with small interaction. It should be emphasized that the assumption about the model form of the Hamiltonian (51) is nonessential for the above derivation. We can start again with the Hamiltonian (51) in which we shall not specify the explicit form of $H_{1}$ and $V$. We assume that the state of the nonequilibrium system is characterized completely by some set of average values $\left\langle P_{k}\right\rangle$ and the state of the thermal bath by $\left\langle H_{2}\right\rangle$. We confine ourselves to such systems for which $\left[H_{1}, P_{k}\right]=\sum_{l} c_{k l} P_{l}$. Then we assume that $\langle V\rangle_{q} \simeq 0$, where $\langle\ldots\rangle_{q}$ denotes the statistical average with the quasi-equilibrium statistical operator of the form

$$
\rho_{q}=Q_{q}^{-1} \exp \left(-\sum_{k} P_{k} F_{k}(t)-\beta H_{2}\right)
$$

and $F_{k}(t)$ are the parameters conjugated with $\left\langle P_{k}\right\rangle$. Following the method used above in the derivation of equation (69), we can obtain the generalized kinetic equations for $\left\langle P_{k}\right\rangle$ with an accuracy up to terms which are quadratic in interaction

$$
\frac{d<P_{k}>}{d t}=\frac{i}{\hbar} \sum_{l} c_{k l}<P_{l}>-\frac{1}{\hbar^{2}} \int_{-\infty}^{0} d t_{1} e^{\varepsilon t_{1}}<\left[\left[P_{k}, V\right], V\left(t_{1}\right)\right]>_{q}
$$

Hence (69) is fulfilled for the general form of the Hamiltonian of a small system weakly coupled to a thermal bath.

\section{SYSTEM IN THERMAL BATH: BALANCE AND MASTER EQUATIONS}

In section [II we have obtained the kinetic equations for $\left\langle P_{\alpha \beta}\right\rangle$ in the general form. Our next task is to write down equations (69) in an explicit form. To do this, we note that the perturbation operator can be represented as $V\left(t_{1}\right)=\sum_{\alpha, \beta} \phi_{\alpha \beta}\left(t_{1}\right) a_{\alpha}^{\dagger} a_{\beta}$, where

$$
\phi_{\alpha \beta}\left(t_{1}\right)=U_{2}\left(t_{1}\right) \Phi_{\alpha \beta} U_{2}^{\dagger}\left(t_{1}\right) \exp \left(\frac{i}{\hbar}\left(E_{\alpha}-E_{\beta}\right) t_{1}\right) ; \quad U_{2}\left(t_{1}\right)=\exp \left(\frac{i H_{2} t_{1}}{\hbar}\right)
$$

Now we calculate the double commutator in the right-hand side of Eq.(69)

$$
\begin{array}{r}
<\left[\left[P_{\alpha \beta}, V\right], V\left(t_{1}\right)\right]>_{q}= \\
\sum_{\mu \nu}\left\{<\Phi_{\beta \mu} \phi_{\mu \nu}\left(t_{1}\right)>_{q}<P_{\alpha \nu}>+<\phi_{\nu \mu}\left(t_{1}\right) \Phi_{\mu \alpha}>_{q}<P_{\nu \beta}>-\left(<\Phi_{\mu \alpha} \phi_{\beta \nu}\left(t_{1}\right)>_{q}+<\phi_{\mu \alpha}\left(t_{1}\right) \Phi_{\beta \nu}>_{q}\right)<P_{\mu \nu}>\right\}
\end{array}
$$

where we restricted ourselves to the linear terms in the mean density of quasiparticles. Let us now remind that the correlation functions $\langle A B(t)\rangle$ and $\langle A(t) B\rangle$ can be expressed via their spectral intensities. Indeed, an effective way of viewing quasiparticles, quite general and consistent, is via the Green functions scheme of many-body theory [68, 78]. It is known [27, 68] that the correlation functions and Green functions are completely determined by the spectral weight function ( or spectral intensity) $J(\omega)$.

$$
\begin{aligned}
& F_{A B}\left(t-t^{\prime}\right)=<A(t) B\left(t^{\prime}\right)>=\frac{1}{2 \pi} \int_{-\infty}^{+\infty} d \omega \exp \left[i \omega\left(t-t^{\prime}\right)\right] J_{A B}(\omega) \\
& F_{B A}\left(t^{\prime}-t\right)=<B\left(t^{\prime}\right) A(t)>=\frac{1}{2 \pi} \int_{-\infty}^{+\infty} d \omega \exp \left[i \omega\left(t^{\prime}-t\right)\right] J_{B A}(\omega)
\end{aligned}
$$

Here the Fourier transforms $J_{A B}(\omega)$ and $J_{B A}(\omega)$ are of the form

$$
\begin{array}{r}
J_{B A}(\omega)= \\
Q^{-1} 2 \pi \sum_{m, n} \exp \left(-\beta E_{n}\right)\left(\psi_{n}^{\dagger} B \psi_{m}\right)\left(\psi_{m}^{\dagger} A \psi_{n}\right) \delta\left(\frac{E_{n}-E_{m}}{\hbar}-\omega\right) \\
J_{A B}(-\omega)=\exp (\beta \hbar \omega) J_{B A}(\omega)
\end{array}
$$

Expressions (76) and (77) are spectral representations of the corresponding time correlation functions. The quantities $J_{A B}$ and $J_{B A}$ are spectral densities or spectral weight functions. 
It is convenient to define

$$
\begin{array}{r}
F_{B A}(0)=<B(t) A(t)>=\frac{1}{2 \pi} \int_{-\infty}^{+\infty} d \omega J(\omega) \\
F_{A B}(0)=<A(t) B(t)>=\frac{1}{2 \pi} \int_{-\infty}^{+\infty} d \omega \exp (\beta \hbar \omega) J(\omega)
\end{array}
$$

The correlation functions $<\Phi_{\beta \mu} \phi_{\mu \nu}\left(t_{1}\right)>_{q}$ and $<\phi_{\nu \mu}\left(t_{1}\right) \Phi_{\mu \alpha}>_{q}$ are connected with their spectral intensities in the following way:

$$
\begin{aligned}
&< \Phi_{\mu \nu} \phi_{\gamma \delta}(t)>_{q}=\frac{1}{2 \pi} \int_{-\infty}^{+\infty} d \omega J_{\gamma \delta, \mu \nu}(\omega) \exp \left[-i\left(\omega-\frac{E_{\gamma}-E_{\delta}}{\hbar}\right) t\right] \\
&<\phi_{\mu \nu}(t) \Phi_{\gamma \delta}>_{q}=\frac{1}{2 \pi} \int_{-\infty}^{+\infty} d \omega J_{\gamma \delta, \mu \nu}(\omega) \exp \left[i\left(\omega+\frac{E_{\mu}-E_{\nu}}{\hbar}\right) t\right]
\end{aligned}
$$

Substituting Eqs. (80) and (81) into Eqs. (69) and (73) and taking into account the notation

$$
\begin{gathered}
\frac{1}{i \hbar} \sum_{\mu} \int_{-\infty}^{0} d t_{1} e^{\varepsilon t_{1}}<\Phi_{\beta \mu} \phi_{\mu \nu}\left(t_{1}\right)>_{q}=\frac{1}{2 \pi} \sum_{\mu} \int_{-\infty}^{+\infty} d \omega \frac{J_{\mu \nu, \beta \mu}(\omega)}{\hbar \omega-E_{\gamma}-E_{\delta}+i \varepsilon}=K_{\beta \nu} \\
\frac{1}{i \hbar} \int_{-\infty}^{0} d t_{1} e^{\varepsilon t_{1}}\left(<\Phi_{\mu \alpha} \phi_{\beta \nu}\left(t_{1}\right)>_{q}+<\phi_{\mu \alpha}\left(t_{1}\right) \Phi_{\beta \nu}>_{q}\right)= \\
\frac{1}{2 \pi} \int_{-\infty}^{+\infty} d \omega J_{\beta \nu, \mu \alpha}(\omega)\left(\frac{1}{\hbar \omega-E_{\beta}+E_{\nu}+i \varepsilon}-\frac{1}{\hbar \omega-E_{\alpha}-E_{\mu}-i \varepsilon}\right)=K_{\alpha \beta, \mu \nu}
\end{gathered}
$$

one can rewrite the kinetic equations for $\left\langle P_{\alpha \beta}>\right.$ as

$$
\frac{d<P_{\alpha \beta}>}{d t}=\frac{1}{i \hbar}\left(E_{\beta}-E_{\alpha}\right)<P_{\alpha \beta}>-\sum_{\nu}\left(K_{\beta \nu}<P_{\alpha \nu}>+K_{\alpha \nu}^{\dagger}<P_{\nu \beta}>\right)+K_{\alpha \beta, \mu \nu}<P_{\mu \nu}>
$$

If one confines himself to the diagonal averages $\left\langle P_{\alpha \alpha}\right\rangle$ only, the last equation may be transformed to give

$$
\begin{gathered}
\frac{d<P_{\alpha \alpha}>}{d t}=\sum_{\nu} K_{\alpha \alpha, \nu \nu}<P_{\nu \nu}>-\left(K_{\alpha \alpha}+K_{\alpha \alpha}^{\dagger}\right)<P_{\alpha \alpha}> \\
K_{\alpha \alpha, \nu \nu}=\frac{1}{\hbar^{2}} J_{\alpha \nu, \nu \alpha}\left(\frac{E_{\alpha}-E_{\beta}}{\hbar}\right)=W_{\beta \rightarrow \alpha} \\
K_{\alpha \alpha}+K_{\alpha \alpha}^{\dagger}=\frac{1}{\hbar^{2}} \sum_{\nu} J_{\nu \alpha, \alpha \nu}\left(\frac{E_{\beta}-E_{\alpha}}{\hbar}\right)=W_{\alpha \rightarrow \beta}
\end{gathered}
$$

Here $W_{\beta \rightarrow \alpha}$ and $W_{\alpha \rightarrow \beta}$ are the transition probabilities expressed in the spectral intensity terms. Using the properties of the spectral intensities [68], it is possible to verify that the transition probabilities satisfy the relation of the detailed balance

$$
\frac{W_{\beta \rightarrow \alpha}}{W_{\alpha \rightarrow \beta}}=\frac{\exp \left(-\beta E_{\alpha}\right)}{\exp \left(-\beta E_{\beta}\right)}
$$

Finally, we have

$$
\frac{d<P_{\alpha \alpha}>}{d t}=\sum_{\nu} W_{\nu \rightarrow \alpha}<P_{\nu \nu}>-\sum_{\nu} W_{\alpha \rightarrow \nu}<P_{\alpha \alpha}>
$$

This equation has the usual form of the Pauli master equation.

According to Ref. [79], "the master equation is an ordinary differential equation, describing the reduced evolution of the system, obtained from the full Heisenberg evolution by taking the partial expectation with respect to the vacuum state of the reservoirs degrees of freedom". The rigorous mathematical derivation of the generalized master equation $[72,73,74,79,80,81,82]$ is rather a complicated mathematical problem. 


\section{A DYNAMICAL SYSTEM IN A THERMAL BATH}

The problem about the appearance of a stochastic process in a dynamical system which is submitted to the influence of a "large" system was considered by Bogoliubov [37, 83]. For a classical system this question was studied on the basis of the Liouville equation for the probability distribution in the phase space and for quantum mechanical systems on the basis of an analogous equation for the von Neumann statistical operator. In the mentioned papers a mathematical method was elaborated which permitted obtaining, in the first approximation, the Fokker-Planck equations. Since then a lot of papers were devoted to studying this problem from various points of view ( e.g. Refs. [84, 85, 86, 87, 88, 89, 90]). Lebowitz and Rubin 84] studied the motion of a Brownian particle in a fluid ( as well as the motion of a Brownian particle in a crystal ) from a dynamical point of view. They derived a formal structure of the collision term similar to the structure of the usual linear transport equation. Kassner 88 used a new type of projection operator and derived homogeneous equations of motion for the reduced density operator of a system coupled to a bath. It was shown that in order to consistently describe damping within quantum mechanics, one must couple the open system of interest to a heat reservoir. The problem of the inclusion of dissipative forces in quantum mechanics is of great interest. There are various approaches to this complicated problem [79, 85, 91, 92, 93]. Tanimura and Kubo 91] considered a test system coupled to a bath system with linear interactions and derived a set of hierarchical equations for the evolution of their reduced density operator. Breuer and Petruccione 92 developed a formulation of quantum statistical ensembles in terms of probability distributions on a projective Hilbert state. They derived a Liouville master equation for the reduced probability distribution of an open quantum system. It was shown that the time-dependent wave function of an open quantum system represented a well-defined stochastic process which is generated by the nonlinear Schrodinger equation

$$
\frac{\partial \psi}{\partial t}=-i G(\psi)
$$

with the nonlinear and non-Hermitian operator $G(\psi)$. The inclusion of dissipative forces in quantum mechanics through the use of non-Hermitian Hamiltonians is of great interest in the theory of interaction between heavy ions. It is clear that if the Hamiltonian has a non-Hermitian part $H_{A}$ the Heisenberg equation of motion will be modified by additional terms. However, care must be taken in defining the probability density operator when the Hamiltonian is non-Hermitian. Also, the state described by the wave function $\psi$ is not then an energy eigenstate because of the energy dissipation. As it was formulated by Accardi and Lu [9], "the quantum Langevin equation is a quantum stochastic differential equation driven by some quantum noise ( creation, annihilation, number noises)." The necessity of considering such processes arises in the description of various quantum phenomena ( e.g., radiation damping, etc.), since quantum systems experience dissipation and fluctuations through interaction with a reservoir 94, 95]. The concept of "quantum noise" was proposed by Senitzky 93. to derive a quantum dissipation mechanism. Originally, the time evolution of quantum systems with the dissipation and fluctuations was described by adding a dissipative term to the quantum equation of motion. However, as was noted by Senitzky [93], this procedure leads to the nonunitary time evolution. He proposed to derive the quantum dissipation mechanism by introducing quantum noise, i.e., a quantum field interacting with the dynamical system (in his case an oscillator). For an appropriately chosen form of the interaction, energy will flow away from the oscillator to the quantum noise field (thermal bath or reservoir).

In this section, we consider the behavior of a small dynamic system interacting with a thermal bath, i.e., with a system that has effectively an infinite number of degrees of freedom, in the approach of the nonequilibrium statistical operator, on the basis of the equations derived in section III The equations derived below can help in the understanding of the origin of irreversible behavior in quantum phenomena.

We assume that the dynamic system ( system of particles) is far from equilibrium with the thermal bath and cannot, in general, be characterized by a temperature. As a result of the interaction with the thermal bath, such a system acquires some statistical characteristics but remains essentially a mechanical system. Our aim is to obtain an equation of evolution ( equations of motion ) for the relevant variables which are characteristic of the system under consideration. The basic idea is to eliminate effectively the thermal bath variables (c.f. Ref. 94, 95, 96] ). The influence of the thermal bath is manifested then as an effect of friction of the particle in a medium. The presence of friction leads to dissipation and, thus, to irreversible processes. In this respect, our philosophy coincides precisely with the Lax statement 94] "that the reservoir can be completely eliminated provided that the frequency shifts and dissipation induced by the reservoir are incorporated into the mean equations of motion, and provided that a suitable operator noise source with the correct moments are added".

Let us consider the behavior of a small subsystem with Hamiltonian $H_{1}$ interacting with a thermal bath with Hamiltonian $\mathrm{H}_{2}$. The total Hamiltonian has the form (51). As operators $P_{m}$ determining the nonequilibrium state of the small subsystem, we take $a_{\alpha}^{\dagger}, a_{\alpha}$, and $n_{\alpha}=a_{\alpha}^{\dagger} a_{\alpha}$. Note that the choice of only the operators $n_{\alpha}$ and $H_{2}$ would lead to kinetic equations (71) for the system in the thermal bath derived above.

The quasi-equilibrium statistical operator (15) is determined from the extremum of the information entropy (17) subject 
to the additional conditions that the quantities

$$
\operatorname{Tr}\left(\rho a_{\alpha}\right)=<a_{\alpha}>, \quad \operatorname{Tr}\left(\rho a_{\alpha}^{\dagger}\right)=<a_{\alpha}^{\dagger}>, \quad \operatorname{Tr}\left(\rho n_{\alpha}\right)=<n_{\alpha}>
$$

remain constant during the variation and the normalization $\operatorname{Tr}(\rho)=1$ is preserved. The operator $\rho_{q}$ has the form

$$
\begin{array}{r}
\rho_{q}=\exp \left(\Omega-\sum_{\alpha}\left(f_{\alpha}(t) a_{\alpha}+f_{\alpha}^{\dagger}(t) a_{\alpha}^{\dagger}+F_{\alpha}(t) n_{\alpha}\right)-\beta H_{2}\right) \equiv \exp (S(t, 0)) \\
\Omega=\ln \operatorname{Tr} \exp \left(-\sum_{\alpha}\left(f_{\alpha}(t) a_{\alpha}+f_{\alpha}^{\dagger}(t) a_{\alpha}^{\dagger}+F_{\alpha}(t) n_{\alpha}\right)-\beta H_{2}\right)
\end{array}
$$

Here, $f_{\alpha}, f_{\alpha}^{\dagger}$ and $F_{\alpha}$ are Lagrangian multipliers determined by the conditions (91). They are the parameters conjugate to $<a_{\alpha}>_{q},<a_{\alpha}^{\dagger}>_{q}$ and $<n_{\alpha}>_{q}$ :

$$
<a_{\alpha}>_{q}=-\frac{\delta \Omega}{\delta f_{\alpha}(t)}, \quad<n_{\alpha}>_{q}=-\frac{\delta \Omega}{\delta F_{\alpha}(t)}, \quad \frac{\delta S}{\delta<a_{\alpha}>_{q}}=f_{\alpha}(t), \quad \frac{\delta S}{\delta<n_{\alpha}>_{q}}=F_{\alpha}(t)
$$

In what follows, it is convenient to write the quasi-equilibrium statistical operator (92) in the form

$$
\rho_{q}=\rho_{1} \rho_{2}
$$

where

$$
\begin{array}{r}
\rho_{1}=\exp \left(\Omega_{1}-\sum_{\alpha}\left(f_{\alpha}(t) a_{\alpha}+f_{\alpha}^{\dagger}(t) a_{\alpha}^{\dagger}+F_{\alpha}(t) n_{\alpha}\right)\right) \\
\Omega_{1}=\ln \operatorname{Tr} \exp \left(-\sum_{\alpha}\left(f_{\alpha}(t) a_{\alpha}+f_{\alpha}^{\dagger}(t) a_{\alpha}^{\dagger}+F_{\alpha}(t) n_{\alpha}\right)\right) \\
\rho_{2}=\exp \left(\Omega_{2}-\beta H_{2}\right), \quad \Omega_{2}=\ln \operatorname{Tr} \exp \left(-\beta H_{2}\right)
\end{array}
$$

The nonequilibrium statistical operator $\rho$ will have the form (23). Note, that the following conditions are satisfied:

$$
<a_{\alpha}>_{q}=<a_{\alpha}>, \quad<a_{\alpha}^{\dagger}>_{q}=<a_{\alpha}^{\dagger}>, \quad<n_{\alpha}>_{q}=<n_{\alpha}>
$$

We shall take, as our starting point, the equations of motion for the operators averaged with the nonequilibrium statistical operator (23)

$$
\begin{aligned}
& i \hbar \frac{d<a_{\alpha}>}{d t}=<\left[a_{\alpha}, H_{1}\right]>+<\left[a_{\alpha}, V\right]> \\
& i \hbar \frac{d<n_{\alpha}>}{d t}=<\left[n_{\alpha}, H_{1}\right]>+<\left[n_{\alpha}, V\right]>
\end{aligned}
$$

The equation for $\left\langle a_{\alpha}^{\dagger}\right\rangle$ can be obtained by taking the conjugate of (98). Restricting ourselves to the second order in the interaction $\mathrm{V}$, we obtain, by analogy with (71), the following equations:

$$
\begin{aligned}
i \hbar \frac{d<a_{\alpha}>}{d t}= & E_{\alpha}<a_{\alpha}>+\frac{1}{i \hbar} \int_{-\infty}^{0} d t_{1} e^{\varepsilon t_{1}}<\left[\left[a_{\alpha}, V\right], V\left(t_{1}\right)\right]>_{q} \\
& i \hbar \frac{d<n_{\alpha}>}{d t}=\frac{1}{i \hbar} \int_{-\infty}^{0} d t_{1} e^{\varepsilon t_{1}}<\left[\left[n_{\alpha}, V\right], V\left(t_{1}\right)\right]>_{q}
\end{aligned}
$$

Here $V\left(t_{1}\right)$ denotes the interaction representation of the operator $V$. Expanding the double commutator in Eq.(100), we obtain

$i \hbar \frac{d<a_{\alpha}>}{d t}=E_{\alpha}<a_{\alpha}>+\frac{1}{i \hbar} \int_{-\infty}^{0} d t_{1} e^{\varepsilon t_{1}}\left(\sum_{\beta \mu \nu}<\Phi_{\alpha \beta} \phi_{\mu \nu}\left(t_{1}\right)>_{q}<a_{\beta} a_{\mu}^{\dagger} a_{\nu}>_{q}-<\phi_{\mu \nu}\left(t_{1}\right) \Phi_{\alpha \beta}>_{q}<a_{\mu}^{\dagger} a_{\nu} a_{\beta}>_{q}\right)$ 
where $\phi_{\mu \nu}\left(t_{1}\right)=\Phi_{\mu \nu}\left(t_{1}\right) \exp \left(\frac{i}{\hbar}\left(E_{\mu}-E_{\nu}\right) t_{1}\right)$. We transform Eq.(102) to

$$
\begin{array}{r}
i \hbar \frac{d<a_{\alpha}>}{d t}=E_{\alpha}<a_{\alpha}>+\frac{1}{i \hbar} \sum_{\beta \mu} \int_{-\infty}^{0} d t_{1} e^{\varepsilon t_{1}}<\Phi_{\alpha \mu} \phi_{\mu \beta}\left(t_{1}\right)>_{q}<a_{\beta}>+ \\
\frac{1}{i \hbar} \sum_{\beta \mu \nu} \int_{-\infty}^{0} d t_{1} e^{\varepsilon t_{1}}<\left[\Phi_{\alpha \nu}, \phi_{\mu \nu}\left(t_{1}\right)\right]>_{q}<a_{\mu}^{\dagger} a_{\nu} a_{\beta}>_{q}
\end{array}
$$

We assume that the terms of higher order than linear can be dropped in Eq.103) ( below, we shall formulate the conditions when this is possible). Then we get

$$
i \hbar \frac{d<a_{\alpha}>}{d t}=E_{\alpha}<a_{\alpha}>+\frac{1}{i \hbar} \sum_{\beta \mu} \int_{-\infty}^{0} d t_{1} e^{\varepsilon t_{1}}<\Phi_{\alpha \mu} \phi_{\mu \beta}\left(t_{1}\right)>_{q}<a_{\beta}>
$$

The form of the linear equation (104) is the same for Bose and Fermi statistics.

Using the spectral representations, Eq.(80) and Eq.(81), it is possible to rewrite Eq.(104) by analogy with Eq.(84) as

$$
i \hbar \frac{d<a_{\alpha}>}{d t}=E_{\alpha}<a_{\alpha}>+\sum_{\beta} K_{\alpha \beta}<a_{\beta}>
$$

where $K_{\alpha \beta}$ is defined in (82). Thus, we have obtained the equation of motion for the average $\left\langle a_{\alpha}\right\rangle$. It is clear that this equation describes approximately the evolution of the state of the dynamic system interacting with the thermal bath. The last term in the right-hand side of this equation leads to the shift of energy $E_{\alpha}$ and to the damping due to the interaction with the thermal bath ( or medium ). In a certain sense, it is possible to say that Eq. (105) is an analog or the generalization of the Schrodinger equation.

Let us now show how, in the case of Bose statistics, we can take into account the nonlinear terms which lead to a coupled system of equations for $\left\langle a_{\alpha}>\right.$ and $\left\langle n_{\alpha}>\right.$. Let us consider the quantity $\left\langle a_{\mu}^{\dagger} a_{\nu} a_{\beta}\right\rangle_{q}$. After the canonical transformation

$$
a_{\alpha}=b_{\alpha}+<a_{\alpha}>, \quad a_{\alpha}^{\dagger}=b_{\alpha}^{\dagger}+<a_{\alpha}^{\dagger}>
$$

the operator $\rho_{1}$ in Eq.(95) can be written in the form

$$
\rho_{1}=Q_{1}^{-1} \exp \left(\Omega_{1}-\sum_{\alpha}\left(F_{\alpha}(t) b_{\alpha}^{\dagger} b_{\alpha}\right)\right), \quad<a_{\alpha}>=-\frac{f_{\alpha}^{\dagger}}{F_{\alpha}}
$$

Note that $Q_{1}$ in (106) is not, in general, equal to $Q_{1}$ in (95). Using the Wick-De Dominicis theorem 68] for the operators $b_{\alpha}^{\dagger}, b_{\alpha}$ and returning to the original operators $a_{\alpha}^{\dagger}, a_{\alpha}$, we obtain

$$
<a_{\mu}^{\dagger} a_{\nu} a_{\beta}>_{q} \simeq\left(<n_{\mu}>-\left|<a_{\mu}>\right|^{2}\right)<a_{\nu}>\delta_{\mu, \beta}+\left(<n_{\mu}>-\left|<a_{\mu}>\right|^{2}\right)<a_{\beta}>\delta_{\mu, \beta}
$$

Using (107), we can rewrite Eq.95) in the form

$$
\begin{array}{r}
i \hbar \frac{d<a_{\alpha}>}{d t}=E_{\alpha}<a_{\alpha}>+\frac{1}{i \hbar} \sum_{\beta \mu} \int_{-\infty}^{0} d t_{1} e^{\varepsilon t_{1}}<\Phi_{\alpha \mu} \phi_{\mu \beta}\left(t_{1}\right)>_{q}<a_{\beta}>+ \\
\frac{1}{i \hbar} \sum_{\mu \beta} \int_{-\infty}^{0} d t_{1} e^{\varepsilon t_{1}}\left(<\left[\Phi_{\alpha \mu}, \phi_{\mu \beta}\left(t_{1}\right)\right]>_{q}+<\left[\Phi_{\alpha \beta}, \phi_{\mu \mu}\left(t_{1}\right)\right]>_{q}\right)\left(<n_{\mu}>+\left|<a_{\mu}>\right|^{2}\right)<a_{\beta}>
\end{array}
$$

Now consider Eq.(101). Expand the double commutator and, in the same way as the threefold terms were neglected in the derivation of Eq.(104), ignore the fourfold terms in (101). We obtain then

$$
\begin{aligned}
& \frac{d<n_{\alpha}>}{d t}=\sum_{\beta} W_{\beta \rightarrow \alpha}\left(<n_{\beta}>+\left|<a_{\beta}>\right|^{2}\right)-\sum_{\beta} W_{\alpha \rightarrow \beta}\left(<n_{\alpha}>+\left|<a_{\alpha}>\right|^{2}\right)+ \\
& \frac{1}{i \hbar} \sum_{\beta} K_{\alpha \beta}<a_{\alpha}^{\dagger}><a_{\beta}>+\frac{1}{i \hbar} \sum_{\beta} K_{\alpha \beta}^{\dagger}<a_{\alpha}><a_{\beta}^{\dagger}>+\sum_{\mu \nu} K_{\alpha \alpha, \mu \nu}<a_{\mu}^{\dagger}><a_{\nu}>
\end{aligned}
$$


Thus, in the general case Eqs.100) and (101) form a coupled system of nonlinear equations of Schrodinger and kinetic types. The nonlinear equation (102) of Schrodinger type is an auxiliary equation and, in conjunction with the equation of kinetic type (109), determines the parameters of the nonequilibrium statistical operator since in the case of Bose statistics

$$
<a_{\alpha}>=-\frac{f_{\alpha}^{\dagger}(t)}{F_{\alpha}(t)}, \quad<n_{\alpha}>=\left(e^{F_{\alpha}(t)}-1\right)^{-1}+\frac{\left|f_{\alpha}\right|^{2}}{F_{\alpha}^{2}(t)}
$$

Therefore, the linear Schrodinger equation is a fairly good approximation if

$$
\left(<n_{\alpha}>+\left|<a_{\alpha}>\right|^{2}\right)=\left(e^{F_{\alpha}(t)}-1\right)^{-1} \ll 1
$$

The last condition corresponds essentially to $<b_{\alpha}^{\dagger} b_{\alpha}>\ll 1$.

In the case of Fermi statistics the situation is more complicated [97|. There is well-known isomorphism between bilinear products of fermion operators and the Pauli spin matrices [98. In quantum field theory the sources linear in the Fermi operators are introduced by means of classical spinor fields that anticommute with one another and with the original field. The Fermion number processes in the time evolution of a certain quantum Hamiltonian model were investigated in Ref. [99]. It was shown that the time evolution tended to the solution of a quantum stochastic differential equation driven by the Fermion number processes. We shall not consider here this complicated case.

In order to interpret the physical meaning of the derived equations, an example will be given here. Let us consider briefly a system of electrons in a lattice described by the Hamiltonian

$$
H=H_{1}+H_{2}+V=\sum_{k \sigma} \epsilon(k) a_{k \sigma}^{\dagger} a_{k \sigma}+\sum_{q} \hbar \omega_{q} b_{q}^{\dagger} b_{q}+\frac{1}{\sqrt{v}} \sum_{k_{1}, k_{2} \sigma} A\left(\overrightarrow{k_{1}}-\overrightarrow{k_{2}}\right) a_{k_{1} \sigma}^{\dagger} a_{k_{2} \sigma}\left(b_{\overrightarrow{k_{1}}-\overrightarrow{k_{2}}}+\overrightarrow{b_{\overrightarrow{k_{2}}}-\overrightarrow{k_{1}}}\right),
$$

where $\hbar \omega_{q}$ is the phonon energy, $a_{k \sigma}^{\dagger}, a_{k \sigma}$ and $b_{q}^{\dagger}, b_{q}$ are the operators of creation and annihilation of electrons and phonons, respectively; $\epsilon(k)$ is the energy of electrons and $A(\vec{q})$ determines the electron-phonon coupling. Equation (105) for $\left.<a_{k \sigma}\right\rangle$ can be represented in the form

$$
i \hbar \frac{d<a_{k \sigma}>}{d t}=(\epsilon(k)+\Delta E(k))<a_{k \sigma}>-\frac{i \hbar}{2} \Gamma(k)<a_{k \sigma}>,
$$

where

$$
\begin{array}{r}
\Delta E(k)=P \sum_{k_{1}}\left|A\left(\vec{k}-\overrightarrow{k_{1}}\right)\right|^{2}\left(\frac{<N_{k-k_{1}}>+1}{\epsilon(k)-\epsilon\left(k_{1}\right)-\hbar \omega_{\vec{k}-\overrightarrow{k_{1}}}}+\frac{<N_{k-k_{1}}>}{\epsilon(k)-\epsilon\left(k_{1}\right)+\hbar \omega_{\vec{k}-\overrightarrow{k_{1}}}}\right)( \\
\Gamma(k)=\frac{2 \pi}{\hbar} \sum_{k_{1}}\left|A\left(\vec{k}-\overrightarrow{k_{1}}\right)\right|^{2}\left(\left(<N_{k-k_{1}}>+1\right) \delta\left(\epsilon(k)-\epsilon\left(k_{1}\right)-\hbar \omega_{\vec{k}-\overrightarrow{k_{1}}}\right)+<N_{k-k_{1}}>\delta\left(\epsilon(k)-\epsilon\left(k_{1}\right)+\hbar \omega_{\vec{k}-\overrightarrow{k_{1}}}\right)\right)(
\end{array}
$$

are the energy shift of an electron and the electron damping, respectively. Here $\left\langle N_{q}>=\left(e^{\beta \hbar \omega_{q}}-1\right)^{-1}\right.$, the distribution functions of the phonons. Expressions (113) and (114) are the same as those obtained by the Green functions method [100] if one sets $<a_{k \sigma}^{\dagger} a_{k \sigma}>\ll 1$ in the latter.

\section{SCHRODINGER-TYPE EQUATION WITH DAMPING FOR A DYNAMICAL SYSTEM IN A THERMAL BATH}

In the previous section we obtained an equation for mean values of the amplitudes in the form (105). It is of interest to analyze and track more closely the analogy with the Schrodinger equation in the coordinate form. To do this, by convention we define the "wave function"

$$
\psi(\vec{r})=\sum_{\alpha} \chi_{\alpha}(\vec{r})<a_{\alpha}>
$$

where $\left\{\chi_{\alpha}(\vec{r})\right\}$ is a complete orthonormalized system of single-particle functions of the operator $\left(-\frac{\hbar^{2}}{2 m} \nabla^{2}+v(\vec{r})\right)$, where $v(\vec{r})$ is the potential energy, and

$$
\left(-\frac{\hbar^{2}}{2 m} \nabla^{2}+v(\vec{r})\right) \chi_{\alpha}(\vec{r})=E_{\alpha} \chi_{\alpha}(\vec{r})
$$


Thus, in a certain sense, the quantity $\psi(\vec{r})$ may plays the role of the wave function of a particle in the medium. Now, using (115), we transform Eq. (105) to

$$
i \hbar \frac{\partial \psi(\vec{r})}{\partial t}=\left(-\frac{\hbar^{2}}{2 m} \nabla^{2}+v(\vec{r})\right) \psi(\vec{r})+\int K\left(\vec{r}, \overrightarrow{r^{\prime}}\right) \psi\left(\overrightarrow{r^{\prime}}\right) d \overrightarrow{r^{\prime}}
$$

The kernel $K\left(\vec{r}, \overrightarrow{r^{\prime}}\right)$ of the integral equation (117) has the form

$$
K\left(\vec{r}, \overrightarrow{r^{\prime}}\right)=\sum_{\alpha \beta} K_{\alpha \beta} \chi_{\alpha}(\vec{r}) \chi_{\beta}^{\dagger}\left(\overrightarrow{r^{\prime}}\right)=\frac{1}{i \hbar} \sum_{\alpha, \beta, \mu} \int_{-\infty}^{0} d t_{1} e^{\varepsilon t_{1}}<\Phi_{\alpha \mu} \phi_{\mu \beta}\left(t_{1}\right)>_{q} \chi_{\alpha}(\vec{r}) \chi_{\beta}^{\dagger}\left(\overrightarrow{r^{\prime}}\right)
$$

Equation (117) can be called a Schrodinger-type equation with damping for a dynamical system in a thermal bath. It is interesting to note that similar Schrodinger equations with a nonlocal interaction are used in the scattering theory [101, 102] to describe interaction with many scattering centers.

To demonstrate the capabilities of equation (117), it is convenient to introduce the operator of translation $\exp (i \vec{q} \vec{p} / \hbar)$, where $\vec{q}=\overrightarrow{r^{\prime}}-\vec{r} ; \vec{p}=-i \hbar \nabla_{r}$. Then Eq. (117) can be rewritten in the form

$$
i \hbar \frac{\partial \psi(\vec{r})}{\partial t}=\left(-\frac{\hbar^{2}}{2 m} \nabla^{2}+v(\vec{r})\right) \psi(\vec{r})+\sum_{p} D(\vec{r}, \vec{p}) \psi(\vec{r})
$$

where

$$
D(\vec{r}, \vec{p})=\int d^{3} q K(\vec{r}, \vec{r}+\vec{q}) e^{\frac{i \vec{q} \vec{p}}{\hbar}}
$$

It is reasonable to assume that the wave function $\psi(\vec{r})$ varies little over the correlation length characteristic of the kernel $K\left(\vec{r}, \overrightarrow{r^{\prime}}\right)$. Then, expanding $\exp (i \overrightarrow{q p} / \hbar)$ in a series, we obtain the following equation in the zeroth order:

$$
i \hbar \frac{\partial \psi(\vec{r})}{\partial t}=\left(-\frac{\hbar^{2}}{2 m} \nabla^{2}+v(\vec{r}+\operatorname{Re} U(\vec{r}))\right) \psi(\vec{r})+i \operatorname{Im} U(\vec{r}) \psi(\vec{r})
$$

where

$$
U(\vec{r})=\operatorname{Re} U(\vec{r})+i \operatorname{Im} U(\vec{r})=\int d^{3} q K(\vec{r}, \vec{r}+\vec{q})
$$

Expression (121) has the form of a Schrodinger equation with a complex potential. Equations of this form are well known in the scattering theory 102$]$ in which one introduces an interaction describing absorption $(\operatorname{Im} U(\vec{r})<0)$. Further, expanding $\exp (i \vec{q} \vec{p} / \hbar)$ in a series up to the second order inclusively, we can represent Eq. (117) in the following form [102]:

$$
i \hbar \frac{\partial \psi(\vec{r})}{\partial t}=\left\{\left(-\frac{\hbar^{2}}{2 m} \nabla^{2}+v(\vec{r})\right)+U(\vec{r})-\frac{1}{i \hbar} \int d^{3} q K(\vec{r}, \vec{r}+\vec{q})(\vec{q} \vec{p})+\frac{1}{2} \int d^{3} q K(\vec{r}, \vec{r}+\vec{q}) \sum_{m, n=1}^{3} q^{m} q^{n} \nabla_{m} \nabla_{n}\right\} \psi(\vec{r})
$$

To interpret this equation, let us introduce the function

$$
\vec{A}(\vec{r})=\frac{m c}{i \hbar e} \int d^{3} q \operatorname{Re} K(\vec{r}, \vec{r}+\vec{q}) \vec{q}
$$

where $m$ and $e$ are the mass and charge of the electron and $c$ is the velocity of light. Then $\vec{A}(\vec{r})$ can be considered, in a certain sense, as an analog of the complex vector potential of an electromagnetic field. It is clear that the motion of a particle (dynamic subsystem) through the medium imitates, to some extent, the motion of a charged particle in the electromagnetic field. To make this analogy even more close, let us introduce the following quantity:

$$
\left(\frac{1}{M(\vec{r})}\right)_{i j}=\frac{1}{m} \delta_{i j}-\frac{m c}{i \hbar e} \int d^{3} q K(\vec{r}, \vec{r}+\vec{q}) q^{i} q^{j}
$$

It follows from (125) that this quantity can be interpreted as a tensor of the reciprocal effective masses 103, 104]. The notion of the "mass tensor" was introduced in [103] to describe the motion of an electron in an external field F

$$
\frac{d v_{i}}{d t}=\frac{e}{\hbar^{2}} \sum_{j} \frac{\partial^{2} E}{\partial q^{i} \partial q^{j}} F_{j} \quad i, j=1,2,3 \quad \text { or } \quad x, y, z
$$


or in vector notation

$$
\frac{d \vec{v}}{d t}=\frac{e}{\hbar^{2}} \operatorname{grad}_{q}\left(\vec{F} \operatorname{grad}_{q} E\right)
$$

Thus, a field $\vec{F}$ may change the velocity $\vec{v}$ in directions other than that of $\vec{F}$. The quantity $\hbar^{2}\left(\frac{\partial^{2} E}{\partial q^{2} \partial q^{j}}\right)^{-1}$ has been called the "mass tensor". Now we can rewrite Eq.(123) in the form

$$
\begin{array}{r}
i \hbar \frac{\partial \psi(\vec{r})}{\partial t}=\left(-\frac{\hbar^{2}}{2} \sum_{i, j=1}^{3}\left(\frac{1}{M(\vec{r})}\right)_{i j} \nabla_{i} \nabla_{j}+v(\vec{r})+U(\vec{r})+\frac{i e \hbar}{m c} \vec{A}(\vec{r}) \vec{\nabla}+i T(\vec{r})\right) \psi(\vec{r}) \\
T(\vec{r})=\frac{1}{2} \int d^{3} q K(\vec{r}, \vec{r}+\vec{q}) \sum_{m, n=1}^{3} q^{m} q^{n} \nabla_{m} \nabla_{n}
\end{array}
$$

Note that in an isotropic medium the tensor $\left(\frac{1}{M(\vec{r})}\right)_{i j}$ is diagonal and $\vec{A}(\vec{r})=0$. The introduction of $\psi(\vec{r})$ does not mean that the state of the small dynamical subsystem is pure. It remains mixed since it is described by the statistical operator (23), the evolution of the parameters $f_{\alpha}, f_{\alpha}^{\dagger}$, and $F_{\alpha}$ of the latter being governed by a coupled system of equations of Schrodinger and kinetic types. It is interesting to mention that the derivation of a Schrodinger-type equation with non-Hermitian Hamiltonian which describes the dynamic and statistical aspects of the motion was declared by Korringa [85]. However, his Eq.(29)

$$
\left.i \frac{\partial W^{\prime}}{\partial t}=\left(H^{\prime}(t)+h^{\prime}(t)\right)+\frac{i}{2 \theta} \frac{d h^{\prime}}{d t}+\ldots\right) W^{\prime}(t)
$$

where $W^{\prime}(t)$ is the statistical matrix for the primed system, can hardly be considered as a Schrodinger-type equation. This special form of the equation for the time-dependent statistical matrix can be considered as a modified Bloch equation.

Hence we were able to apply the NSO approach given above to dynamics. We have shown in this section that for some class of dynamic systems it was possible, with the NSO approach, to go from a Hamiltonian description of dynamics to a description in terms of processes which incorporates the dissipativity. However, a careful examination is required in order to see under what conditions the Schrodinger-type equation with damping can really be used.

\section{CONCLUDING REMARKS}

In this paper, we have discussed the general statistical mechanics approach to the description of the transport processes. We have applied the method of the nonequilibrium statistical operator to study the generalized kinetic and evolution equations. We analyzed and derived in a closed form the kinetic equations and applied them to some typical problems.

In writing the paper we have essentially confined ourselves to a discussion of those features of the theory which deal with general structural properties rather than with specific physical applications. The method offers several advantages over the standard technique of the calculation of transport coefficients. The derived generalized kinetic equations for a system weakly coupled to a thermal bath are analogous to those derived in [52] for the system of weakly interacting particles. Moreover, the capability of the generalized kinetic equations was demonstrated and further discussed by considering a few representative examples, i.e., the kinetic equations for magnons and phonons, and the energy shift and damping of particle (electron) due to the friction with media (phonons). There are many other applications of the formalism developed in this article, for example, longitudinal nuclear spin relaxation and spin diffusion. However, we have not considered other contributions here. These questions deserve a separate consideration.

An example of a small system being initially far from equilibrium has been considered. We have reformulated the theory of the time evolution of a small dynamic system weakly coupled to a thermal bath and shown that a Schrodingertype equation emerges from this theory as a particular case. Clearly then, the nonequilibrium statistical operator approach is a convenient and workable tool for the derivation of relaxation equations and formulae for evolution and kinetic equations.

In our above treatment we have avoided a number of important questions such as the rigorous proof of the existence and uniqueness of the quasi-equilibrium state, the validity of the time-smoothing procedure, etc. These questions, as well as the application of the derived equations to other important problems of transport in solids such as the nuclear 
spin relaxation and diffusion, electro- and thermal conductivity, remain to be areas for further investigation.

[1] N. N. Bogoliubov and J. N. N. Bogoliubov, Introduction to Quantum Statistical Mechanics (World Scientific, Singapore, 1982).

[2] D. Chandler, Introduction to Modern Statistical Mechanics (Oxford University Press, Oxford, 1988).

[3] W. T. Grandy, Foundations of Statistical Mechanics: Equilibrium Theory, vol. 1 (D. Reidel Publ., Doddrecht, Holland, 1987).

[4] W. T. Grandy, Foundations of Statistical Mechanics: Nonequilibrium Phenomena, vol. 2 (D. Reidel Publ., Doddrecht, Holland, 1988).

[5] M. Toda, R. Kubo, and N. Saito, Statistical Physics: Equilibrium Statistical Mechanics, vol. 1 (Springer-Verlag, Berlin, New York, 1992).

[6] R. Kubo, M. Toda, and N. Hashitsume, Statistical Physics: Nonequilibrium Statistical Mechanics, vol. 2 (Springer-Verlag, Berlin, New York, 1991).

[7] D. A. McQuarrie, Statistical Mechanics (University Science Books, California, 2000).

[8] L. Boltzmann, Lectures on Gas Theory (Univ. of Califrnia Press, Berkeley, 1964).

[9] R. Peierls, in Transport Phenomena, edited by G. Kirczenow and J. Marro (Springer, Berlin, 1974), vol. 31 of Lecture Notes in Physics, p. 1.

[10] G. E. Uhlenbeck, in Rarefied Gas Dynamics (Academic Press, New York, 1974), p. 3.

[11] J. W. Gibbs, Elementary Principles in Statistical Mechanics (Dover Publ., New York, 1960).

[12] M. S. Green, J. Chem. Phys. 20, 1281 (1954).

[13] M. S. Green, J. Chem. Phys. 22, 398 (1954).

[14] E. W. Montroll and M. S. Green, in Annual Review of Physical Chemistry (J. Wiley and Sons, New York, 1954), vol. 5, p. 449.

[15] H. Mori, J. Phys. Soc. Jpn. 11, 102 (1956).

[16] H. Mori, Phys. Rev. 111, 694 (1958).

[17] H. Mori, Phys. Rev. 112, 1829 (1958).

[18] H. Mori, Phys. Rev. 115, 298 (1959).

[19] R. Kubo, J. Phys. Soc. Jpn. 12, 570 (1957).

[20] R. Kubo, M. Yokota, and S. Nakajima, J. Phys. Soc. Jpn. 12, 1203 (1957).

[21] S. Nakajima, Prog. Theor. Phys. 20, 948 (1958).

[22] D. N. Zubarev, Doklady Akademii Nauk SSSR 140, 92 (1961).

[23] I. Prigogine, Nonequilibrium Statistical Mechanics (Interscience Publ., New York, 1962).

[24] H. Mori, Prog. Theor. Phys. 33, 423 (1965).

[25] H. Mori, Prog. Theor. Phys. 34, 399 (1965).

[26] M. H. Lee, Phys. Rev. E 61, 3571 (2000).

[27] D. N. Zubarev, Nonequilibrium Statistical Thermodynamics (Consultant Bureau, New York, 1974).

[28] J. A. McLennan, Introduction to Nonequilibrium Statistical Mechanics (Prentice Hall, New Jersey, 1989).

[29] M. Ichiyanagi, Phys. Rep. 243, 125 (1994).

[30] R. Zwanzig, Nonequilibrium Statistical Mechanics (Oxford University Press, Oxford, 2001).

[31] J. L. Lebowitz, Physica A 263, 516 (1999).

[32] E. W. Montroll, in Termodinamica dei Processi Irreversibili, edited by S. DeGroot (Societa Italiana di Fizica, Bologna, 1960), vol. X, p. 217.

[33] D. N. Zubarev, Teor. Mat. Fiz. 3, 276 (1970).

[34] A. Carati and L. Galgani, Physica A 280, 106 (2000).

[35] R. M. Lewis, Journal Math. Phys. 8, 1448 (1967).

[36] N. N. Bogoliubov, Problems of a Dynamical Theory in Statistical Physics (in Russian) (State Techn. Publ., Moscow, 1946).

[37] N. N. Bogoliubov, Communications E17-10514, JINR, Dubna (1977).

[38] P. G. Bergmann and J. L. Lebowitz, Phys. Rev. 99, 578 (1955).

[39] J. L. Lebowitz and P. G. Bergmann, Ann. Phys. 1, 1 (1957).

[40] J. L. Lebowitz and A. Shimony, Phys. Rev. 128, 1945 (1962).

[41] B. Robertson, Phys. Rev. 144, 151 (1966).

[42] B. Robertson, Phys. Rev. 160, 175 (1967).

[43] B. Robertson, Journal Math. Phys. 11, 2482 (1970).

[44] B. Robertson and W. C. Mitchell, Journal Math. Phys. 12, 563 (1971).

[45] V. P. Kalashnikov and D. N. Zubarev, Physica 59, 314 (1972).

[46] Z. Onyszkiewicz, Physica A 143, 287 (1987).

[47] M. Gell-Mann and M. L. Goldberger, Phys. Rev. 91, 398 (1953).

[48] M. Goldberger and K. Watson, Collision Theory (J. Wiley and Sons, New York, 1964).

[49] P. V. Coveney, Nature 333, 409 (1988). 
[50] W. E. Lamb, in Quantum Statistical Mechanics in Natural Sciences, edited by B. Kursunogly, S. L. Mintz, and S. M. Wildmayer (Plenum Press, New York, 1969), p. 213.

[51] N. N. Bogoliubov, Communications D-781, JINR, Dubna (1961).

[52] L. A. Pokrovsky, Doklady Akademii Nauk SSSR 183, 806 (1968).

[53] L. Reggiani, Hot-Electron Transport in Semiconductors (Springer, Berlin, 1985).

[54] M. Sparks, Ferromagnetic-Relaxation Theory (McGraw-Hill Book, New York, 1964).

[55] R. Peierls, Quantum Theory of Solids (Clarendon Press, Oxford, 1955).

[56] V. P. Kalashnikov, Physica 48, 93 (1970).

[57] D. Y. Xing, P. Hu, and C. S. Ting, Phys. Rev. B 35, 6379 (1987).

[58] E. Schlomann, Phys. Rev. 121, 1312 (1961).

[59] T. G. Phillips and H. M. Rosenberg, Rep. Prog. Phys. 29, 285 (1966).

[60] P. Carruthers, Rev. Mod. Phys. 33, 92 (1961).

[61] J. J. Green and E. Schlomann, J. Appl. Phys. 32, 168 (1961).

[62] J. J. Green and E. Schlomann, J. Appl. Phys. 33, 1358 (1962).

[63] E. Schlomann and J. J. Green, J. Appl. Phys. 34, 1291 (1963).

[64] R. I. Joseph, E. Schlomann, and R. M. White, J. Appl. Phys. 34, 2686 (1963).

[65] R. I. Joseph, C. P. Hartwig, T. Kohane, and E. Schlomann, J. Appl. Phys. 37, 1069 (1966).

[66] R. I. Joseph and E. Schlomann, J. Appl. Phys. 38, 1915 (1967).

[67] E. Schlomann, Phys. Rev. 182, 632 (1969).

[68] S. V. Tyablikov, Methods in the Quantum Theory of Magnetism (Plenum Press, New York, 1967).

[69] S. Lepri, R. Livi, and A. Politi, Phys. Rep. 377, 1 (2003).

[70] R. Peierls, Ann. Physik (Leipzig) 3, 1055 (1929).

[71] G. P. DeVault and J. A. McLennan, Phys. Rev. 138, 856 (1965).

[72] L. van Hove, Physica 23, 441 (1957).

[73] B. Leaf, Journal Math. Phys. 11, 1806 (1970).

[74] R. Zwanzig, Physica 30, 1109 (1964).

[75] G. V. Chester, Rep. Prog. Phys. 26, 411 (1963).

[76] J. L. Lebowitz, Phys. Rev. 114, 1192 (1959).

[77] E. A. Jackson, J. R. Pasta, and J. F. Waters, J. Comput. Phys. 2, 207 (1968).

[78] A. L. Kuzemsky, Rivista Nuovo Cimento 25, 1 (2002).

[79] L. Accardi and Y. G. Lu, Commun. Math. Phys. 141, 9 (1991).

[80] R. J. Swenson, Journal Math. Phys. 3, 1017 (1962).

[81] R. L. Peterson and P. M. Quay, Journal Math. Phys. 5, 85 (1964).

[82] A. Fulinski and W. J. Kramarczyk, Physica 39, 575 (1968).

[83] N. N. Bogoliubov and N. M. Krylov, in Proc. Dept. Math. Phys. Acad. Sci. Ukraine (Acad. Sci. Ukraine, Kiev, 1939), vol. 4 , p. 5 .

[84] J. L. Lebowitz and E. Rubin, Phys. Rev. 131, 2381 (1963).

[85] J. Korringa, Phys. Rev. 133, A1228 (1964).

[86] L. Galgani and G. LoVecchio, Nuovo Cimento B 52, 1 (1979).

[87] G.Benettin, L. Galgani, and A. Giogilli, Nature 311, 444 (1984).

[88] K. Kassner, Phys. Rev. A 36, 5381 (1987).

[89] V. Romero-Rochin and I. Oppenheim, Physica A 155, 52 (1989).

[90] A. Carati and L. Galgani, Phys. Rev. E 61, 4791 (2000).

[91] Y. Tanimura and R. Kubo, J. Phys. Soc. Jpn. 58, 101 (1989).

[92] H. P. Breuer and F. Petruccione, Phys. Rev. E 51, 4041 (1995).

[93] I. R. Senitzky, Phys. Rev. 119, 670 (1960).

[94] M. Lax, Phys. Rev. 145, 110 (1966).

[95] M. Lax and W. H. Louisell, Phys. Rev. 185, 568 (1969).

[96] G. Chanmugam and S. S. Schweber, Phys. Rev. A 1, 1369 (1970).

[97] F. A. Berezin, The Method of Second Quantization (Academic Press, New York, 1966).

[98] F. A. Kaempfer, Concepts in Quantum Mechanics (Academic Press, New York, 1965).

[99] L. Accardi and Y. G. Lu, Ann. Inst. Henry Poincare 58, 127 (1993).

[100] D. N. Zubarev, Uspekhi. Fiz. Nauk 71, 71 (1960).

[101] M. Lax, Rev. Mod. Phys. 23, 287 (1951).

[102] N. F. Mott and H. S. Massey, The Theory of Atomic Collisions (Clarendon Press, Oxford, 1965).

[103] D. I. Blochinzev and L. Nordheim, Z. Physik 84, 168 (1933).

[104] N. F. Mott and H. Jones, The Theory of the Properties of Metals and Alloys (Dover Publ., New York, 1968). 